\title{
Transcription factor TBX4 regulates myofibroblast accumulation and lung fibrosis
}

\author{
Ting Xie, ${ }^{1}$ Jiurong Liang, ${ }^{1}$ Ningshan Liu, ${ }^{1}$ Caijuan Huan, ${ }^{1,2}$ Yanli Zhang, ${ }^{1,3}$ Weijia Liu, ${ }^{4,5}$ Maya Kumar, ${ }^{6}$ Rui Xiao, ${ }^{7}$ Jeanine D'Armiento, ${ }^{7}$ \\ Daniel Metzger, ${ }^{8}$ Pierre Chambon, ${ }^{8}$ Virginia E. Papaioannou, ${ }^{9}$ Barry R. Stripp, ${ }^{1}$ Dianhua Jiang, ${ }^{1}$ and Paul W. Noble ${ }^{1}$ \\ 'Department of Medicine and the Women's Guild Lung Institute, Cedars-Sinai Medical Center, Los Angeles, California, USA. ${ }^{2}$ Department of Respiratory Medicine, The First Affiliated Hospital, \\ Zhejiang University, Hangzhou, China. Institute of Basic Medical Sciences, Chinese Academy of Medical Sciences, Beijing, China. ${ }^{4}$ Department of Medicine, Duke University School of Medicine, \\ Durham, North Carolina, USA. ${ }^{5}$ Department of Respiratory Medicine, People's Hospital of Guizhou Province, Guiyang, Guizhou, China. ${ }^{6}$ Department of Biochemistry and Howard Hughes Medical Institute, \\ Stanford University School of Medicine, Stanford, California, USA. 'Department of Anesthesiology and Physiology and Cellular Biophysics, Columbia University Medical Center, New York, \\ New York, USA. IInstitut de Cénétique et de Biologie Moléculaire et Cellulaire, CNRS UMR7104, Inserm U964, Strasbourg, France. ${ }^{9}$ Department of Cenetics and Development, \\ Columbia University Medical Center, New York, New York, USA.
}

\begin{abstract}
Progressive tissue fibrosis is a major cause of the morbidity and mortality associated with repeated epithelial injuries and accumulation of myofibroblasts. Successful treatment options are limited by an incomplete understanding of the molecular mechanisms that regulate myofibroblast accumulation. Here, we employed in vivo lineage tracing and real-time gene expression transgenic reporting methods to analyze the early embryonic transcription factor T-box gene 4 (TBX4), and determined that TBX4-lineage mesenchymal progenitors are the predominant source of myofibroblasts in injured adult lung. In a murine model, ablation of TBX4-expressing cells or disruption of TBX4 signaling attenuated lung fibrosis after bleomycin-induced injury. Furthermore, TBX4 regulated hyaluronan synthase 2 production to enable fibroblast invasion of matrix both in murine models and in fibroblasts from patients with severe pulmonary fibrosis. These data identify TBX4 as a mesenchymal transcription factor that drives accumulation of myofibroblasts and the development of lung fibrosis. Targeting TBX4 and downstream factors that regulate fibroblast invasiveness could lead to therapeutic approaches in lung fibrosis.
\end{abstract}

\section{Introduction}

Progressive tissue fibrosis is a major cause of morbidity and mortality with limited therapeutic choices. Severe pulmonary fibrosis is the integrative result of repeated epithelial injuries and recruitment and activation of myofibroblasts, as well as matrix deposition. Idiopathic pulmonary fibrosis (IPF) is a terminal illness characterized by unremitting matrix deposition in the lung (1). The accumulation of myofibroblasts is one of the key features in tissue fibrosis.

The origins of myofibroblasts have been of great interest in understanding the pathogenesis of tissue fibrosis (2-5). Fibroblasts in IPF (6) and in mouse models (5) are extremely heterogeneous, suggesting that they could be derived from different cell types or represent different stages of activation, or that they may be influenced by the surrounding milieu (7). Identification of fibroblast-specific cell surface markers has been elusive (7). Markers such as $\alpha$-smooth muscle actin ( $\alpha$ SMA, encoded by the Acta2 gene), FSP1 (also known as S100A4), vimentin, desmin, and PDGFR $\beta$ are either not exclusively expressed by fibroblasts or not specific to all fibroblasts $(5,8)$. Recent reports suggest that several cellular sources contribute to myofibroblasts. Cells of extrapulmonary origin may be able to migrate to the fibrotic lesion and become myofibroblasts (9-11). Several studies have proposed that epithelial cells $(12-14)$ or endothelial cells $(4,15,16)$ transform into stro-

Conflict of interest: The authors have declared that no conflict of interest exists. Submitted: October 30, 2015; Accepted: May 12, 2016.

Reference information: / Clin Invest. 2016;126(8):3063-3079. doi:10.1172/JCI85328. mal cells in experimental fibrosis models. However, using genetic tracing approaches, recent studies have shown that lung epithelial cells such as adult $S f t p c$-lineage alveolar type II cells, as well as adult Scgb1a1-lineage club cells, do not give rise to fibroblasts (5). In addition, genetic lineage labeling studies have confirmed that pericytes trace-labeled with either NG2, FOXJ1, or FOXD1 (3, 5) contribute to $\alpha$ SMA-expressing myofibroblasts. Finally, GLI1labeled perivascular cells with distinct characteristics of mesenchymal stem cells (MSCs) can differentiate into myofibroblasts in tissue fibrosis (17). These studies suggest that a number of cell types may contribute to myofibroblast accumulation, but key upstream regulatory molecules that may promote myofibroblast accumulation from a number of cellular origins have not been identified. In particular, we sought to determine whether there might be a transcription factor that promotes myofibroblast accumulation from different cells of origin.

T-box gene 4 (TBX4) is a mesenchymal-specific transcription factor that is expressed early during embryonic development and is essential for hindlimb development $(18,19)$ and airway branching (20). TBX4 expression is detected in lung buds at E9.25 (20), and TBX4 is expressed at E11.5 and later throughout the lung mesenchyme (20). GLI1 is detectable from E11.5, and FOXD1 from E13.5, both of which are later than TBX4 $(3,21)$. These reports raise the possibility that TBX4 might be upstream of other mesenchymal transcription factors that regulate the diversity of mesenchymal progenitor populations with different locations, patterns of migration, and recruitment mechanisms. Mice with both TBX4 
and TBX5 deficiency exhibit severely reduced lung branching at mid-gestation, indicating that TBX4 is important in regulating the proliferation, migration, and differentiation of mesenchymal cells that support and help the generation of surrounding epithelium (20). We sought to determine whether TBX4 is playing a role in disease contexts such as fibrosis in the adult lung. In addition, we investigated the role of TBX4 in regulating key effector functions of fibroblasts such as proliferation, migration, and invasion as well as myofibroblast accumulation, activation, and extracellular matrix (ECM) gene expression in vitro and in vivo.

Through comprehensive analyses including tracking of mesenchymal markers in vivo, multicolor clonal cell labeling, cell-specific lineage ablation, and gene deletion in a cell type-specific fashion, we show here that Tbx4-derived cells give rise to a diversity of cell types including fibroblasts, smooth muscle cells, some pericytes, and a few endothelial cells in the adult lung. In addition, Tbx4-lineage cells expand, are proliferative, and form clonal-like patches within fibrotic foci. Furthermore, Tbx4-derived cells are major contributors to myofibroblast accumulation. Specific deletion of Tbx4-lineage cells or the Tbx4 gene in either collagen- or aSMA-expressing fibroblasts significantly attenuated lung fibrosis. Lastly, TBX4 binding sites in the hyaluronan synthase 2 (Has2) promoter regulate expression and enhance fibroblast invasiveness and hyaluronan (HA) production, revealing the importance of this pathway in fibrogenesis. Collectively, these data suggest that TBX4 may be an important regulator of fibrogenic potential of diverse cell types that contribute to myofibroblast accumulation.

\section{Results}

Tbx4-lineage cells give rise to fibroblasts, smooth muscle, and rare endothelial cells in the lung. To permanently label TBX4-expressing cells and their descendants in the lung, Tbx4 lung enhancer-Cre transgenic mice $\left(T b x 4^{L M E}-C r e\right.$, also known as $\left.T b x 4-C r e^{T g}\right)$ (22) or Tbx4-Cre knock-in mice $\left(T b x 4-C r e^{K i}\right)(23,24)$ were crossed with Rosa26-tdTomato (Tm) reporter mice (25). It has been shown that TBX4 is expressed as early as E9.25 in lung mesenchyme (20). We found that $T b x 4$ lineage-labeled stromal cells reside along blood vessels, underneath the bronchioles, and within the interstitial mesenchyme, of both embryonic (E15.5) and adult-stage (8W) lung tissue (Figure 1, A-C; and Supplemental Figure 1B; supplemental material available online with this article; doi:10.1172/ JCI85328DS1). Tbx4-lineage cells include lipofibroblasts and a few endothelial cells (Figure 1, G and H). These results are consistent with recent data indicating that Tbx4-Cre labels undifferentiated mesenchyme, airway smooth muscle, vascular smooth muscle, and mesothelium $(22,26,27)$. The locations of tdTomato-labeled (tdT-labeled) cells were identical in the lungs of both strains.

To determine whether Tbx4-lineage cells expanded during injury, adult Tbx $4^{L M E}$-Cre Rosa26-tdTomato and Tbx4-Cre ${ }^{K i}$ Rosa26-tdTomato mice were administered $2.5 \mathrm{U} / \mathrm{kg}$ bleomycin intratracheally, and the lungs were examined 21 days after injury (Figure 1A and Supplemental Figure 1A). We found extensive expansion of Tbx4-derived cells on day 21 in comparison with saline-treated lung (Figure 1, D and E, and Supplemental Figure 1B).

We then costained with stromal markers and demonstrated overlap of Tbx4-lineage cells with several stromal cell markers, including SMA, desmin, vimentin, collagen type $1 \alpha 1$ (COL1 1 1), and pericyte markers PDGFR $\beta$ and NG2 (Figure $1 \mathrm{~F}$ ) in the lungs of Tbx $4^{L M E}$-Cre Rosa26-tdTomato mice. Overlap of tdT labeling and aSMA staining can be easily seen in airway smooth muscle, vascular smooth muscle, and interstitial fibroblasts (Figure $1 \mathrm{~F}$ ). Overlap of $\mathrm{tdT}^{+}$labeling and staining of fibroblast markers (COL1 $\alpha 1$, desmin, and vimentin) can also be readily identified, especially in the lungs 21 days after bleomycin injury (Figure $1 F$ ). The antibody staining results were similar in the lungs of both $T b x 4^{L M E_{-}} \mathrm{Cre}$ Rosa26-tdTomato and Tbx4-Cre ${ }^{K i}$ Rosa26-tdTomato mice.

Pericytes have been implicated in tissue fibrosis and are suggested to be a source of myofibroblasts $(3,28,29)$. PDGFR $\beta$ and chondroitin sulfate proteoglycan 4 (CSPG4, or NG2), along with FOXD1 and FOXJ1, have each been used as pericyte markers (3, 5). In Ng2-YFP Tbx4-Cre ${ }^{K i}$ Rosa26-tdTomato triple-heterozygous mice, there were $0.40 \%$ and $0.88 \%$ NG2/tdT double-positive cells within total cells in uninjured and bleomycin-injured (day 21) lung single-cell homogenates, respectively (Supplemental Figure 2B). A few $\mathrm{NG}^{+}$cells are of $\mathrm{Tbx} 4$ lineage in both bleomycininjured (22\%) and uninjured (10\%) Ng2-YFP Tbx4-Cre ${ }^{K i}$ Rosa26tdTomato mouse lungs (Supplemental Figure 2C). Within $\mathrm{tdT}^{+}$ cells, $10 \%$ were $\mathrm{NG}^{+}$in both uninjured and bleomycin-injured lungs (Supplemental Figure 2C).

Epithelial cells defined by their expression of SCGB1A1, SFTPC, T1a (Figure 1I), or E-cadherin (not shown) were absent from the tdT-labeled fraction of lung cells. This is consistent with previous studies in embryonic lungs that also indicate that Tbx4lineage cells do not include an epithelial subset $(22,24)$.

Tbx4-lineage cells were rarely observed in extrapulmonary tissues of either Tbx $4^{L M E}$-Cre Rosa26-tdTomato or Tbx4-Cre $e^{K i}$ Rosa26-tdTomato mice. In bone marrow, we found that only a very small fraction (less than $0.03 \%$ ) of the total live cells expressing tdT in Tbx4-Cre ${ }^{K i}$ Rosa26-tdTomato mice, whereas no Tbx4-lineage cells were detected in Tbx $4^{L M E}$-Cre Rosa26-tdTomato bone marrow (Supplemental Figure 3). Other organs of 8-week-old Tbx4-Cre ${ }^{K i}$ Rosa26-tdTomato mice, including heart, liver, kidney, spleen, and intestine, were all negative for Tbx4-lineage cells (data not shown).

Next, we wanted to determine whether Tbx4-lineage cells give rise to MSCs. GLI1-labeled perivascular cells with distinct characteristics of MSCs can differentiate into myofibroblasts in tissue fibrosis (17). Our data suggest that Tbx4-lineage cells do not include cells expressing a classic MSC pattern $\left(\mathrm{CD} 44^{+} \mathrm{CD} 29^{+} \mathrm{CD} 105^{+} \mathrm{SCA} 1^{+}\right.$ and $\left.\mathrm{CD} 31^{-} \mathrm{CD} 34^{-} \mathrm{CD} 45^{-}\right)$, although about $70 \%$ of $\mathrm{Tb} \times 4$-lineage cells express CD29 (integrin- $\beta_{1}$ ) (Supplemental Figure 4).

We next compared the expression profiles of $\mathrm{tdT}^{+}$versus $\operatorname{tdT}^{-}$cells, which were sorted from cultured lung fibroblasts of Tbx $4^{L M E}$-Cre Rosa26-tdTomato mice. Genes that were expressed at higher levels in $\mathrm{tdT}^{+}$cells included fibroblast-related genes and ECM remodeling genes such as Acta2, Adam12, Pdgfra, Fgf1O, and Fgf7, genes in the collagen family (Col1a2, Col3a1, Col4a1, Col4a2, Col4a5, Col4a6), Mmps (Mmp10, Mmp11, Mmp8), desmin, and Has2 (Supplemental Table 1). In addition, several transcription factors including SOX11, Smad genes (Smad1, Smad2, Smad3, Smad5, and Smad7), Snai1, Snai2, and T-box genes (Tbx2, Tbx3, $T b x 5, T b x 18$, and of course $T b x 4$ itself) were also upregulated in $\operatorname{tdT}^{+}$cells (Supplemental Table 2). These data suggest that Tbx4lineage cells express more fibroblast- and ECM remodelingrelated genes indicative of a more activated state. 
A

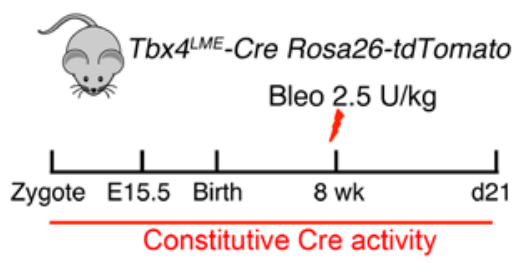

B

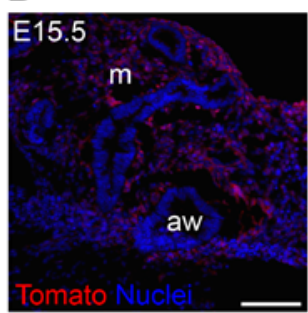

C

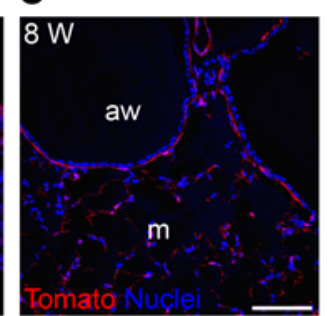

D

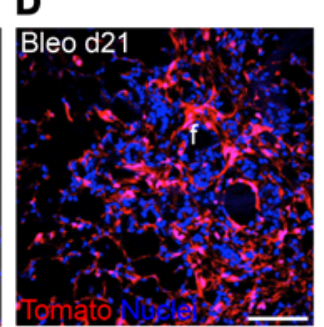

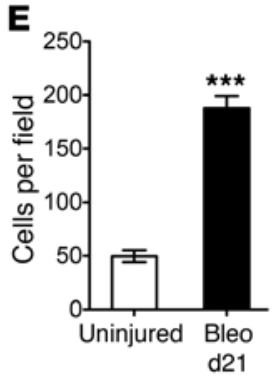

F
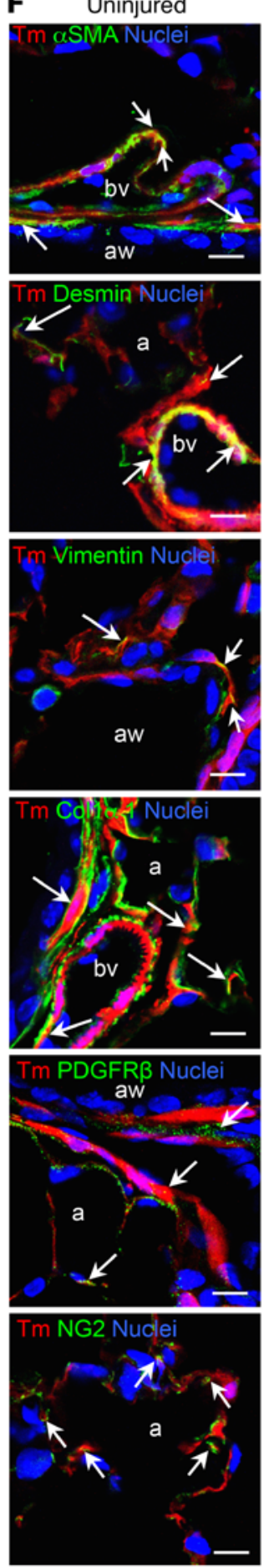

Bleo d21
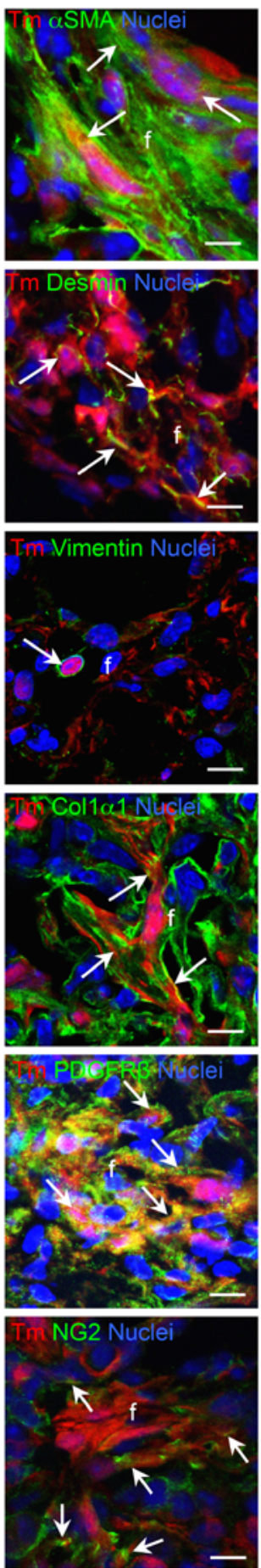

G Uninjured

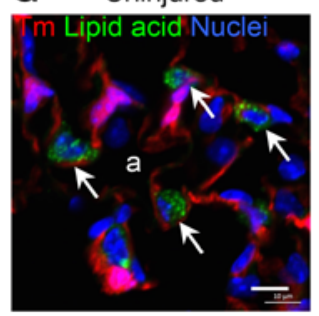

H

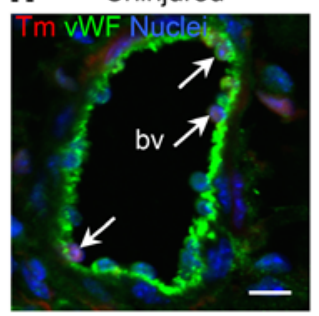

I Uninjured
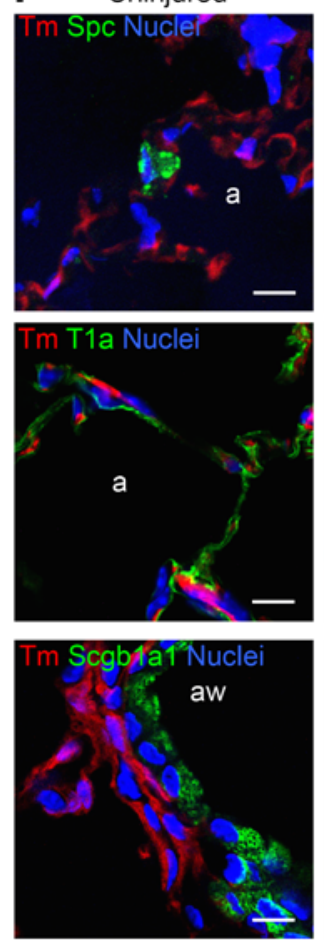

Bleo d21

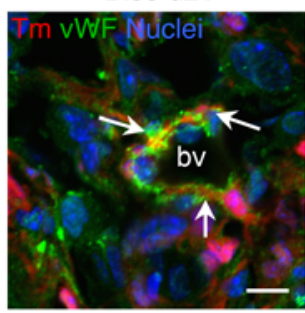

Bleo d21
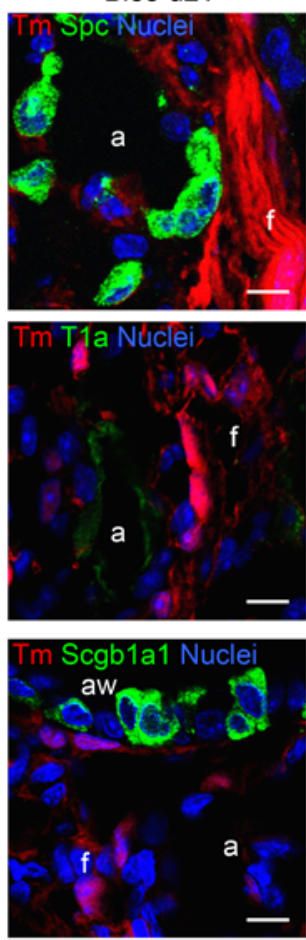

Figure 1. Tbx4-lineage cells include smooth muscle cells, fibroblasts, some pericytes, and endothelial cells in both normal and injured lung. (A) Schematic depicting lineage analysis methodology. Tbx4 ${ }^{\llcorner M E}$-Cre Rosa26-tdTomato mice were used for all experiments in this figure. (B-D) Representative histological sections of Tbx $4^{\text {LME }_{-}}$ Cre Rosa26-tdTomato mouse lungs at embryonic d15.5 (B) and uninjured 8-week-old (C) and bleomycin-treated (d21) (D) adult Tbx4 ${ }^{{ }^{M E}}$-Cre Rosa26-tdTomato with Tbx4-lineage cells in red and nuclei in blue. (E) Quantification of Tbx4 lineage tracing, expressed as cells counted in $\mathbf{C}$ and $\mathbf{D}$ ( $n=9$ in each group of Tbx $4^{\text {LME-Cre Rosa26- }}$ tdTomato mice; ${ }^{* *} P \leq 0.001$ by 2-tailed Student's $t$ test; mean \pm SEM). (F-I) Representative immunofluorescent images of adult Tbx $4^{L M E}$-Cre Rosa26-tdTomato mouse lungs, uninjured or bleomycin-treated (d21) and stained with $\alpha S M A$, desmin, vimentin, COL1 $\alpha 1$, PDGFR $\beta$, NG2 (F), lipid acid (G), vWF (H), SPC, T1a, and SCGB1A1 (I) antibody ( $n=9$ lungs examined). Arrows show cells with overlaps. Scale bars: $100 \mu \mathrm{m}$ (B-D) and $10 \mu \mathrm{m}$ (F-I). a, alveoli; aw, airway; bv, blood vessel; $\mathrm{f}$, fibrotic foci; $\mathrm{m}$, mesenchyme; Tomato and Tm, tdTomato. 
A
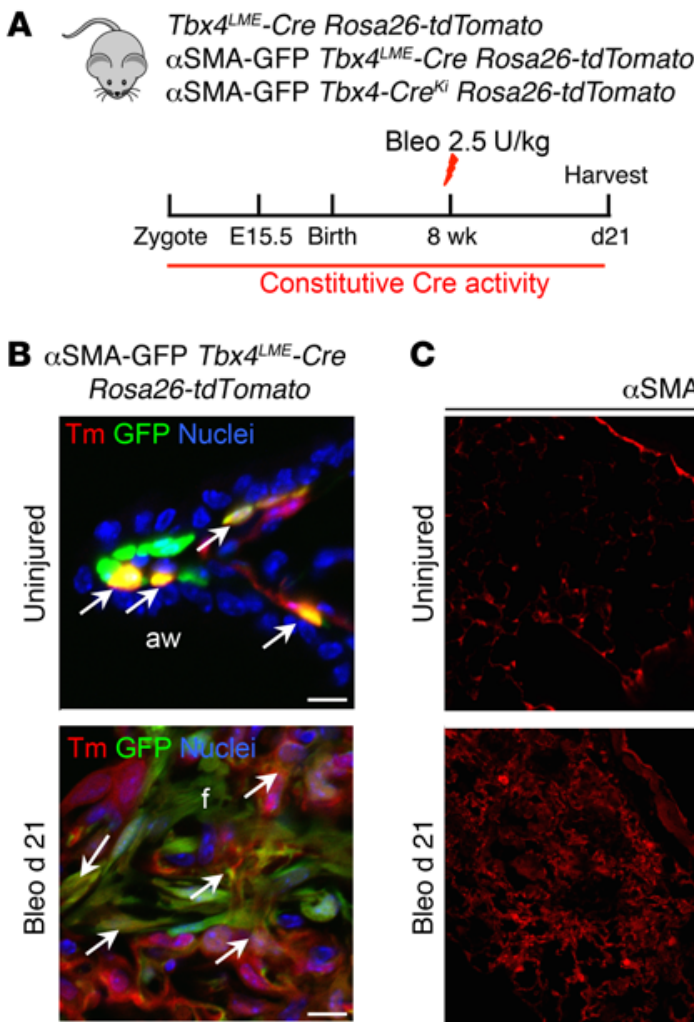

C
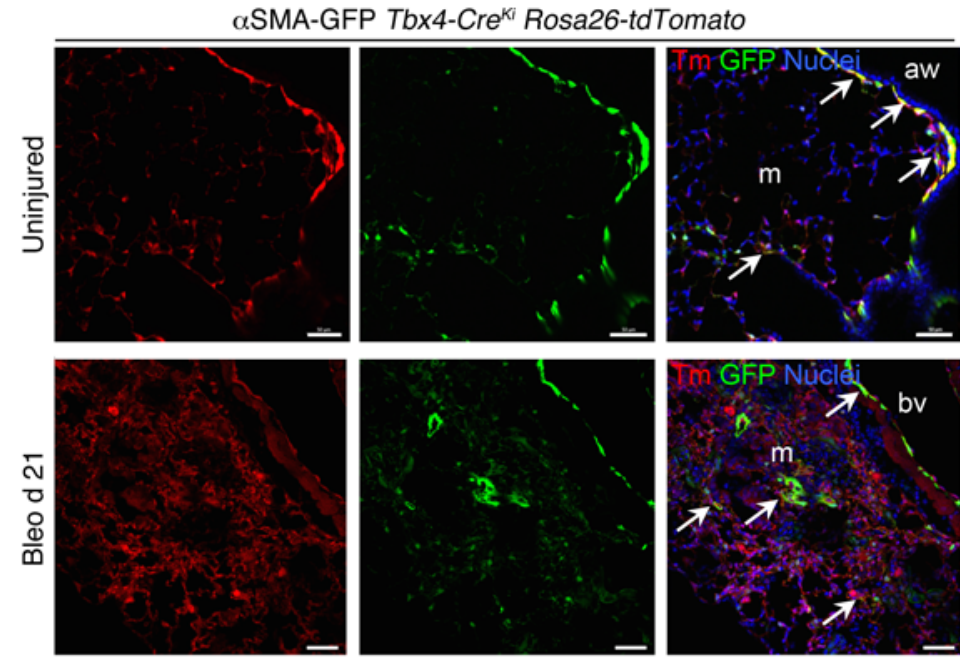

D
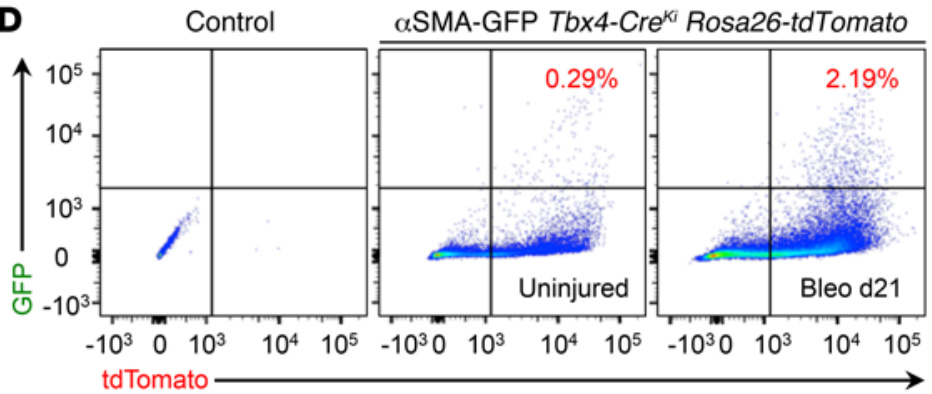

E

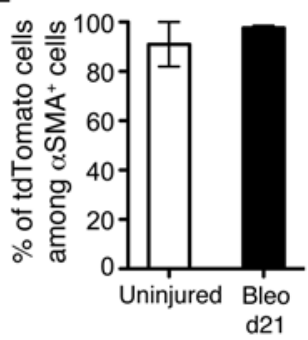

Figure 2. Tbx4-lineage cells in the lung are the major source of myofibroblasts. (A) Schematic depicting lineage analysis methodology. Tbx4-Cre ${ }^{L M E}$ Rosa26-tdTomato, aSMA-GFP Tbx4 ${ }^{L M E}$-Cre Rosa26-tdTomato, and $\alpha$ SMA-GFP Tbx4-Cre ${ }^{K i}$ Rosa26-tdTomato mice were used for all experiments in this figure. (B) Representative histology images of uninjured or bleomycin-treated (d21) adult $\alpha$ SMA-GFP Tbx $4^{\text {LME}_{-}-\mathrm{Cre}}$ Rosa26-tdTomato mouse lung. (C) Representative histology images of uninjured or bleomycin-treated (d21) adult $\alpha$ SMA-GFP Tbx4-Cre ${ }^{K i}$ Rosa26-tdTomato mouse lung. (D) Representative FACS plots of mouse lung single cells isolated from uninjured or bleomycin-treated (d21) QSMA-GFP Tbx4-Cre ${ }^{K i}$ Rosa26-tdTomato mice. (E) Quantification of tdT ${ }^{+}$ cells among $\alpha$ SMA-GFP ${ }^{+}$cells in D $(n=3$ mice analyzed, mean \pm SEM). Arrows show cells with overlaps. Scale bars: $10 \mu \mathrm{m}$ (B) and $100 \mu \mathrm{m}$ (C). aw, airway; bv, blood vessel; f, fibrotic foci; $m$, mesenchyme.
TGF- $\beta$ signaling is a prominent driver in tissue fibrosis (30). It is has been reported that conditional deletion of TGF- $\beta$ receptor II in COL1 $\alpha 2-$ and TBX4-expressing cells significantly reduces lung fibrosis $(31,32)$. We found that $\mathrm{TBX} 4^{+}$fibroblasts have an activated phenotype and express higher levels of Smad transcription factors (Supplemental Table 2). We wondered whether the activated phenotype in TBX $4^{+}$cells was dependent on TGF- $\beta$ signaling. To our surprise, exogenous TGF- $\beta$ did not increase Tbx4 gene expression in either mouse or human fibroblast cultures (Supplemental Figure 5, A-E). In our array data, both TGF- $\beta 1$ and TGF- $\beta R 1$ expression was decreased in $\mathrm{TBX} 4^{+}$fibroblasts compared with TBX4- fibroblasts. However, both TGF- $\beta 2$ and TGF$\beta \mathrm{R} 2$ expression was elevated in $\mathrm{TBX} 4^{+}$fibroblasts compared with TBX4- fibroblasts (Supplemental Table 3). Interestingly, we found that the TGF- $\beta$ superfamily member BMP 4 can induce the expression of Tbx4 mRNAs in cultured primary human lung fibroblasts. Similar results were found following WNT5A treatment (Supplemental Figure 5F). These data suggested a complex role for TBX 4 in TGF- $\beta$ signaling; further investigations are needed to elucidate the relationship.
Together, these results indicate that Tbx4-lineage cells in the lung represent a heterogeneous cell population that includes smooth muscle cells and different types of fibroblasts, as well as some pericytes and endothelial cells. Tbx4-lineage fibroblasts expand during pulmonary fibrosis and significantly contribute to $\alpha \mathrm{SMA}^{+}$myofibroblast accumulation.

The vast majority of myofibroblasts derive from Tbx4-lineage cells. To further confirm the $\alpha$ SMA antibody staining results, we crossed the reporter mouse line Acta2-GFP (referred to here as aSMAGFP mice) with Tbx $4^{L M E}-$ Cre Rosa26-tdTomato and Tbx4-Cre ${ }^{K i}$ Rosa26-tdTomato mice. FACS analysis of lung single cells from uninjured aSMA-GFP Tbx4-Cre ${ }^{K i}$ Rosa26-tdTomato mice revealed that approximately $80 \%$ of the $7 \mathrm{AAD}^{-}, \mathrm{EPCAM}^{-}$, and $\mathrm{CD} 31^{-} \mathrm{CD} 45^{-}$ cells are Tbx4-lineage cells (Supplemental Figure 6). The histology images of $\alpha$ SMA-GFP Tbx $4^{L M E}$-Cre Rosa26-tdTomato and $\alpha \mathrm{SMA}$ GFP Tbx4-Cre ${ }^{K i}$ Rosa26-tdTomato mouse frozen sections are consistent with the antibody staining results (Figure 2, B and C). The Acta2 promoter drives GFP expression well in the lung, as the expression pattern and the levels were comparable with antibody staining (Supplemental Figure 7). In uninjured aSMA-GFP Tbx4- 
A

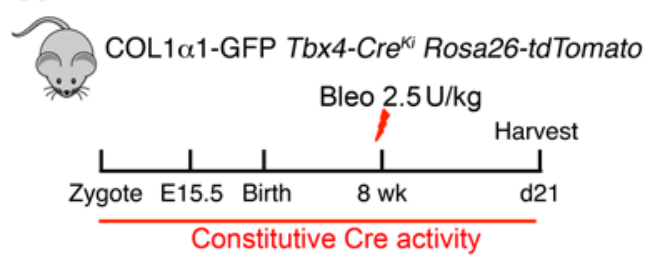

B

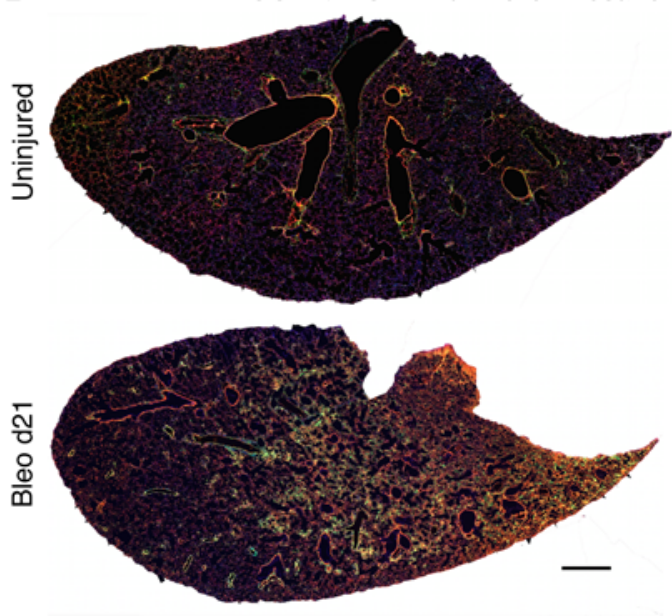

COL1a1-GFP Tbx4-Cre ${ }^{k_{i}}$ Rosa26-tdTomato
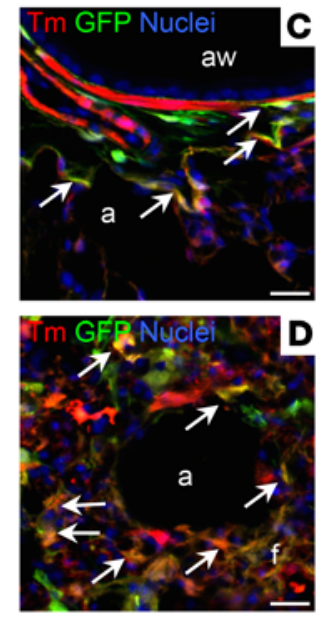

$\mathbf{E}$

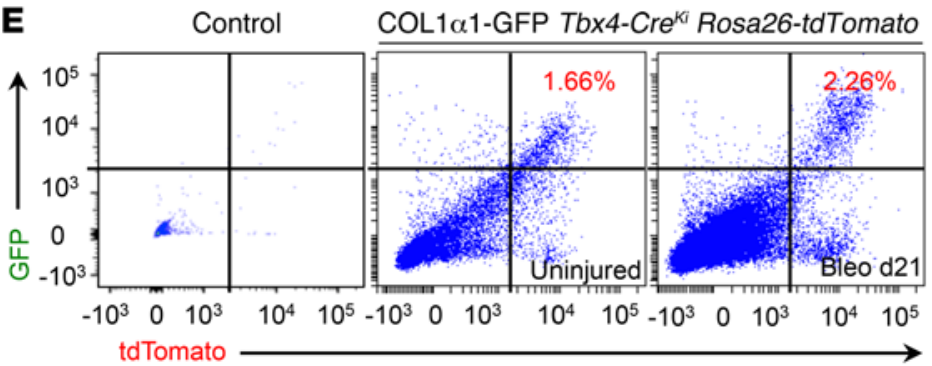

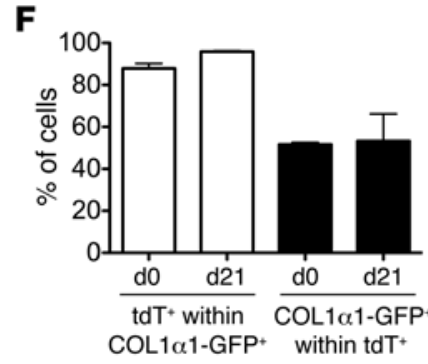

Figure 3. Tbx4-lineage cells in the lung are the major source of COL1 $11^{+}$fibroblasts. (A) Schematic depicting lineage analysis methodology. COL1 1 1-GFP Tbx4-Cre ${ }^{K i}$ Rosa26-tdTomato mice were used for all experiments in this figure. (B-D) Representative histology images of uninjured or bleomycin-treated (d21) adult COL1a1-GFP Tbx4-Cre ${ }^{K i}$ Rosa26-tdTomato mouse lung. (E) Representative FACS plots of mouse lung single cells isolated from uninjured or ble-

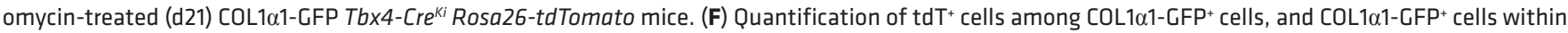
$\mathrm{tdT}^{+}$cells in $\mathbf{E}(n=3$ mice analyzed, mean \pm SEM). Arrows show cells with overlaps. Scale bars: $1 \mathrm{~mm}$ (B) and $10 \mu \mathrm{m}$ (C and $\mathbf{D})$. a, alveoli; aw, airway.

Cre ${ }^{K i}$ Rosa26-tdTomato triple-heterozygous mice, $0.29 \%$ of total cells in lung single-cell homogenates were $\alpha \mathrm{SMA} / \mathrm{tdT}$ double-positive cells as determined by flow cytometric analysis (Figure 2D). The SMA/tdT double-positive cells increased more than 7-fold to $2.19 \%$ of total cells, in the lungs of mice 21 days after bleomycin exposure (Figure 2D). Among $\alpha \mathrm{SMA}-\mathrm{GFP}^{+}$lung cells, $90.6 \%$ were $\mathrm{tdT}^{+}$in uninjured mice, and $97.6 \%$ were $\mathrm{tdT}^{+} 21$ days after bleomycin exposure (Figure 2E). These data confirmed that most myofibroblasts were derived from $T b x 4$ lineage-labeled resident lung stromal cells.

The majority of COL1 $\alpha 1^{+}$cells are derived from Tbx4-lineage cells. COL1 $\alpha 1$-expressing cells are interstitial mesenchymal cells and fibrocytes in tissues $(15,33)$. The reporter mouse line Col1a1-GFP (referred to here as COL1 $\alpha 1-G F P$ mice) (33) was crossed with Tbx4$\mathrm{Cre}^{\mathrm{Ki}}$ Rosa26-tdTomato mice to generate COL1a1-GFP Tbx4$\mathrm{Cre}^{\mathrm{Ki}}$ Rosa26-tdTomato triple-heterozygous mice. FACS analysis of lung single cells from uninjured COL1a1-GFP Tbx4-Cre ${ }^{K i}$ Rosa26-tdTomato mice revealed that more than $80 \%$ of the $7 \mathrm{AAD}^{-}$, $\mathrm{EPCAM}^{-}$, and $\mathrm{CD}^{-} \mathrm{CD}^{-} 5^{-}$cells are $\mathrm{Tb} x 4$-lineage cells (Supplemental Figure 8). The mice were subjected to intratracheal bleomycin-induced lung injury (Figure 3A). Analysis of frozen lung section at day 21 after bleomycin showed that most of the COL1 $\alpha 1-$ $\mathrm{GFP}^{+}$cells were labeled by mesenchymal-specific Tbx4-Cre (Figure $3, \mathrm{~B}-\mathrm{D})$, suggesting that COL1 $\alpha 1$-expressing fibroblasts were predominantly derived from Tbx4-lineage mesenchymal cells. Following bleomycin-induced lung fibrosis, COL1 $\alpha 1$-expressing and $T b x 4$-lineage fibroblast numbers were visibly increased. Flow cytometry analysis showed that $1.16 \%$ and $2.26 \%$ of the total lung single cells were COL1 $\alpha 1 /$ tdT double-positive in uninjured and bleomycin-injured (day 21) lungs, respectively (Figure 3E). Moreover, $87 \%$ of all $\mathrm{GFP}^{+}$cells were $\mathrm{tdT}^{+}$in uninjured lungs, and $95 \%$ of total GFP cells were $\mathrm{tdT}^{+}$in bleomycin-injured lungs. Among $\mathrm{tdT}^{+}$cells, $50 \%$ of the cells are $\mathrm{GFP}^{+}$in both uninjured and bleomycin-injured lungs (Figure 3F). Together, these results demonstrate that the majority of COL1 $\alpha 1$-expressing fibroblasts are $T b x 4$ lineage-labeled cells and the Tbx4-derived population is a larger population than COL $1 \alpha 1^{+}$cells.

TBX4-expressing cells proliferate and expand during injury. We next sought to determine the role of TBX4-expressing cells in regulating fibroblast accumulation in the adult mice. To address this question, we carried out tamoxifen-induced cell lineage tracing experiments to specifically label postnatal TBX4-expressing cell types. Tbx $4^{L M E_{-}}$CreER transgenic mice (22) were bred with Rosa26-tdTomato mice, allowing tamoxifen-regulated tdT labeling of the TBX4-expressing cells (Figure 4A).

Tamoxifen dose-dependent increases were observed in the percentage of tdT-labeled cells in adult $T b x 4^{L M E}$-CreER Rosa26tdTomato mice (Supplemental Figure 9A). Mice were then 
A

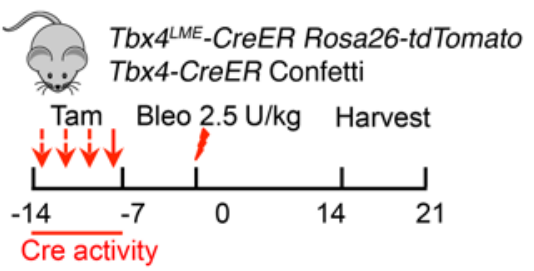

$\mathbf{F}$



Uninjured
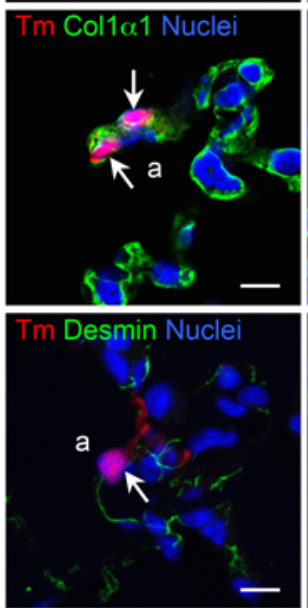
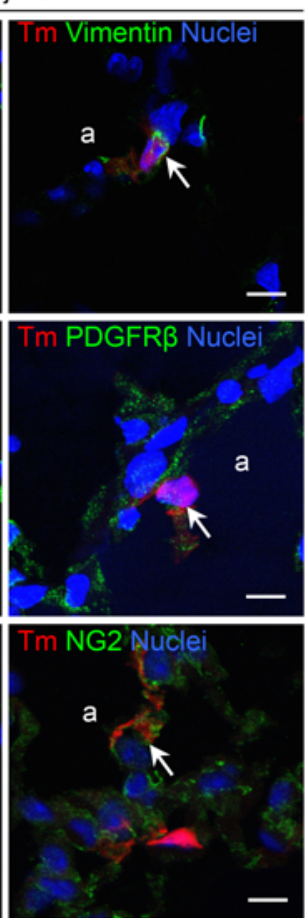
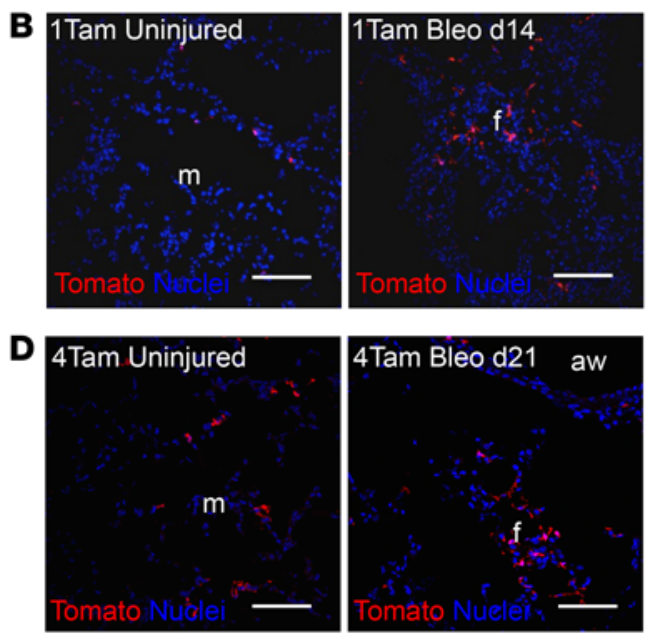

Bleo d21
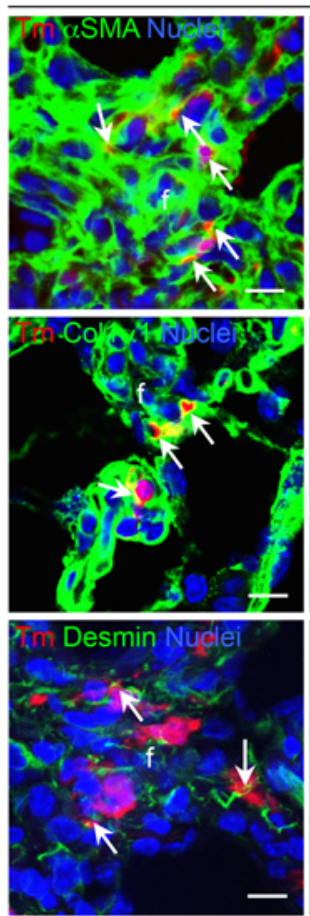
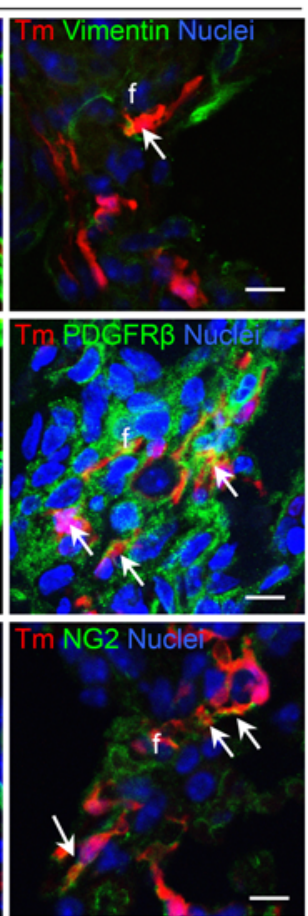
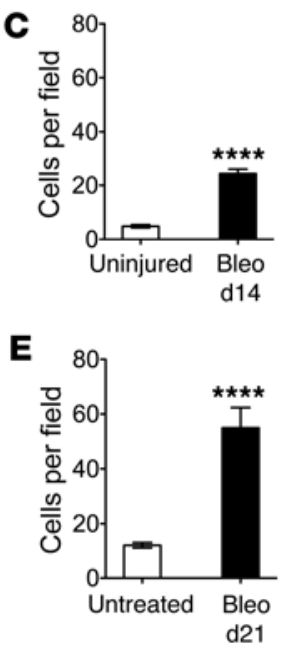

G

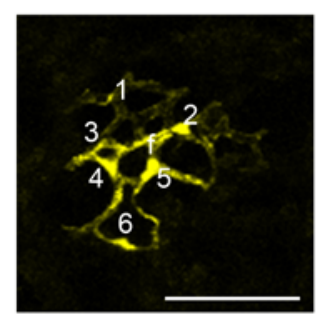

H

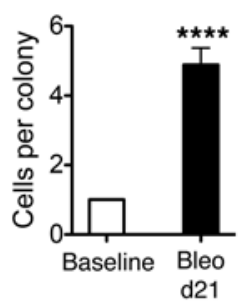

Figure 4. Tbx4-lineage cells are highly proliferative upon bleomycin injury in the lung. (A) Experimental design for inducible TBX4 cell labeling using 1 or 4 doses of tamoxifen 1 week before bleomycin injury for Tbx4 ${ }^{\text {LME }}$-CreER Rosa26-tdTomato mice. Lungs were harvested on d21. Tbx4-Cre ${ }^{K i}$ Rosa26-tdTomato (Tam, $0.2 \mathrm{mg} / \mathrm{g} /$ dose) and Tbx4 ${ }^{L M E}-$ CreER Confetti (Tam, $0.1 \mathrm{mg} / \mathrm{g} /$ dose) were used for all experiments in this figure. (B and D) Representative lung sections from Tbx4 ${ }^{L M E}$-CreER Rosa26-tdTomato mice with 1 (B) or 4 (D) doses of tamoxifen injection showing Tbx4-lineage cells in red and nuclei in blue. (C and E) Quantification of Tbx4 cells counted in B and $\mathbf{D}$ (from 2 lobes per mouse, $n=9$ mice; ${ }^{* * *} P \leq 0.0001$ by 2-tailed Student's $t$ test; mean \pm SEM). (F) Representative immunofluorescent images of mice from B staining with $\alpha$ SMA, COL1 $\alpha 1$, desmin, vimentin, PDCFR 3 , and NG2. Arrows show cells with overlap in staining ( $n=6$ lungs examined). (G) TBX4 cells were marked in Tbx $4^{L M E}$-CreER Confetti mice using 4 doses of tamoxifen. Representative confocal images of a typical colony of TBX4 cells. Cells in clone are enumerated. (H) TBX4 colony size at baseline and d21 after bleomycin injury. $n=9$ lungs examined. ${ }^{* * * *} P \leq 0.0001$ by Student's $t$ test; mean \pm SEM. Scale bars: $100 \mu \mathrm{m}$ (B and $\mathbf{D}$ ) and $10 \mu \mathrm{m}$ (F and G). a, alveoli; aw, airway; f, fibrotic foci; m, mesenchyme.

given either 1 or 4 doses of tamoxifen 1 week before bleomycin injury. Lung tissue was harvested for histology at both 14 and 21 days after bleomycin administration (Figure 4A). We detected a significant increase in $\mathrm{tdT}^{+}$cells in mouse lungs at both 14 and 21 days after bleomycin exposure compared with lungs from untreated control mice (Figure $4, \mathrm{~A}-\mathrm{E}$ ). These $\mathrm{tdT}^{+}$cells costained with $\alpha \mathrm{SMA}, \mathrm{COL} 1 \alpha 1$, desmin, vimentin, PDGFR $\beta$,
NG2, and lipid acid, as well as vWF antibodies (Figure 4F and Supplemental Figure 9, C and D). However, $\operatorname{tdT}^{+}$cells did not costain with SPC, T1a, or SCGB1A1 (Supplemental Figure 9E). Additionally, BrdU labeling at 21 days indicated that TBX4expressing cells lineage-labeled before injury were proliferating 21 days after bleomycin exposure of Tbx $4^{L M E}$-CreER Rosa26tdTomato mice (Supplemental Figure 9, F and G). 
A

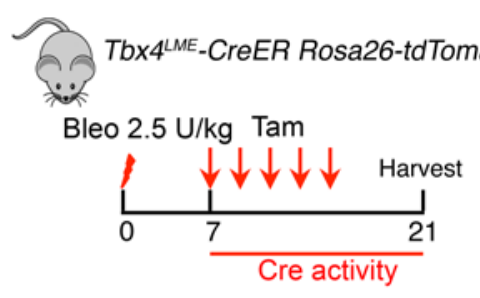

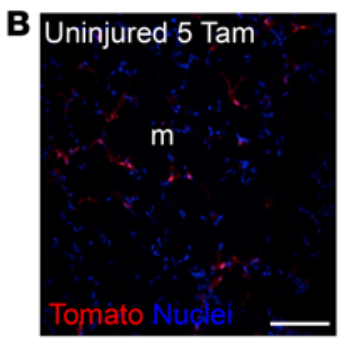

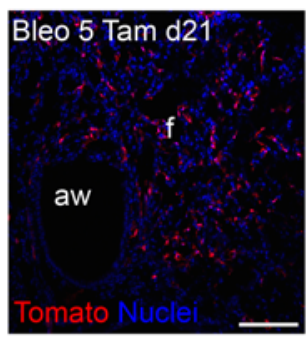

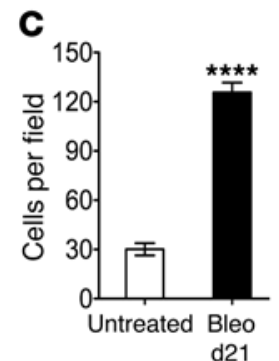

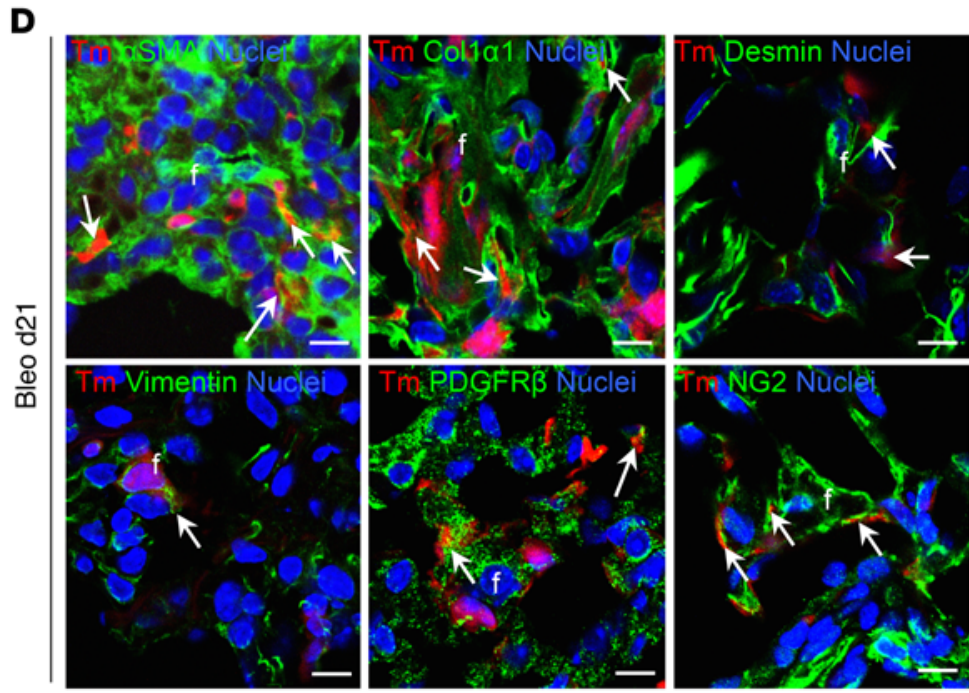

Figure 5. TBX4 cells are induced de novo upon bleomycin injury in the lung. (A) Five doses of tamoxifen ( 0.2 $\mathrm{mg} / \mathrm{g} /$ dose) were given to Tbx $4^{\text {LME-CreER Rosa26- }}$ $t d$ Tomato every other day starting from d7 after bleomycin treatment. Lungs were harvested for histology on d21 after bleomycin. (B) Representative lung sections from Tbx $4^{\text {LME }}$-CreER Rosa26-tdTomato mice in A showing Tbx4-lineage cells in red and nuclei in blue. (C) Quantification of TBX4 cells in B (from 2 lobes per mouse, $n=9$ mice; ${ }^{* * *} P \leq 0.0001$ by Student's $t$ test; mean \pm SEM). (D) Representative images of $\alpha S M A$, COL1 $\alpha 1$, desmin, vimentin, PDGFR $\beta$, and NG2 immunofluorescence staining. Arrows show the overlap cells. Scale bars: $100 \mu \mathrm{m}$ (B) and $10 \mu \mathrm{m}$ (D). aw, airway; f, fibrotic foci; m, mesenchyme.

Next, we crossed Tbx $4^{L M E}$-CreER mice with Confetti mice (34) to determine whether Tbx4-lineage cells are clonally expanded (Figure 4A). In light of the low frequency of lineage labeling within lung tissue, patches of adjacent lineage-labeled cells harboring a common fluorescent protein tag were considered to be clones and to reflect the clonal behavior of TBX4-expressing progenitors. Analysis of clone size as a function of time following bleomycin exposure indicated that $T b x 4$-lineage cells underwent progressive clonal expansion after bleomycin exposure (Figure 4, G and $\mathrm{H}$, and Supplemental Figure 10). These data demonstrate that Tbx4-lineage fibroblastic progenitors undergo clonal expansion during lung injury and fibrosis. We therefore conclude that TBX4expressing cells labeled before injury proliferated and expanded.

Tbx4-lineage cells are induced de novo by injury. In order to test whether TBX4 expression is induced de novo by injury, we carried out experiments by labeling TBX4-expressing cells by tamoxifen after injury. We administered 5 doses of tamoxifen every other day beginning 1 week following bleomycin injection in Tbx $4^{\mathrm{LME}_{-}-C r e E R}$ Rosa26-tdTomato mice, and the lungs were harvested on day 21 (Figure 5A). We observed a significant increase of $\mathrm{tdT}^{+}$cells in bleomycin-treated mice compared with untreated control mice (Figure 5, B and C). The progeny of TBX4-expressing cells in bleomycin-treated mice costained with aSMA, COL1 $\alpha 1$, desmin, vimentin, PDGFR $\beta$, and NG2 (Figure 5D). Hence, these data suggest that TBX4 is induced de novo after injury and TBX4-expressing cells labeled after injury contributed to the expansion of Tbx4-lineage cells. TBX4 expression is high in the embryonic mouse lungs $(24,26)$ and is much lower in adult mouse lungs $(22,26)$. These data suggest that TBX4 signaling can be re-activated under pathological conditions such as tissue injury.

Ablation of TBX4-expressing cells inhibits lung fibrosis. To directly demonstrate the functional role of TBX4-expressing fibroblasts and their cellular progeny in lung fibrosis, $T b x 4^{L M E}-$ CreER mice were crossed with Rosa26-Stop-DTA or Rosa26-tdTomato Rosa26-Stop-DTA mice, in which the tamoxifen-inducible, Cre-mediated combination will result in deletion of TBX4-expressing cells. These mice were given 5 doses of tamoxifen for 1 week to induce the ablation of TBX4-expressing cells (Figure 6A). As expected, injection of tamoxifen induced a marked reduction of the progeny of TBX 4 cells ( $\mathrm{tdT}^{+}$cells) (Figure 6, B and C), of Tbx4 and Has 2 mRNA, and of HA production in lung fibroblasts (Figure 6, D-F). To determine whether there are any structural changes in Tbx4-lineage cell-ablated lungs at baseline, we performed H\&E staining, and mean linear intercept calculation to assess for emphysema. Our data suggest that there are no significant structural changes in either uninjured or relatively normal areas of injured lung with short-term TBX4 cell deletion (Supplemental Figure 11, A and B). We detected a significant decrease in collagen content $(\sim 61 \%)$ in tamoxifeninjected Tbx $4^{L M E}$-CreER Rosa26-DTA mouse lungs 21 days after bleomycin by Masson's trichrome staining (Figure 7, A-G) and hydroxyproline assay (Figure $7 \mathrm{H}$ ). Immunofluorescence analysis also revealed decreasing of COL1 $\alpha 1, \alpha \mathrm{SMA}$, and HA expression (Figure 7, I-K). These data indicate that Tbx4-lineage cells play a critical role in regulating fibrosis in the bleomycin-induced lung injury model. Furthermore, the loss of TBX4-expressing cells and their progeny attenuated interstitial lung fibrosis. 
A

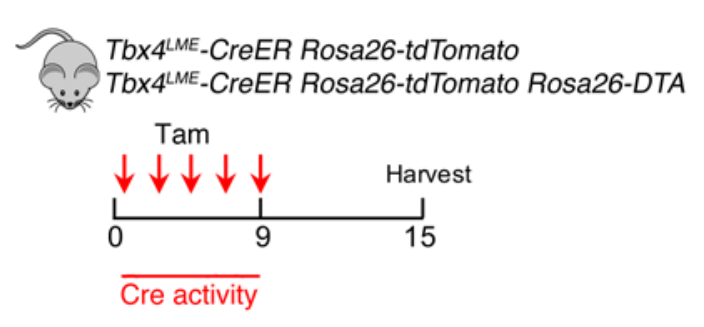

B

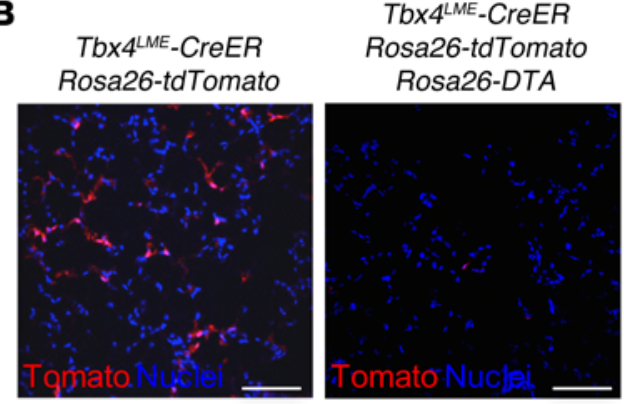

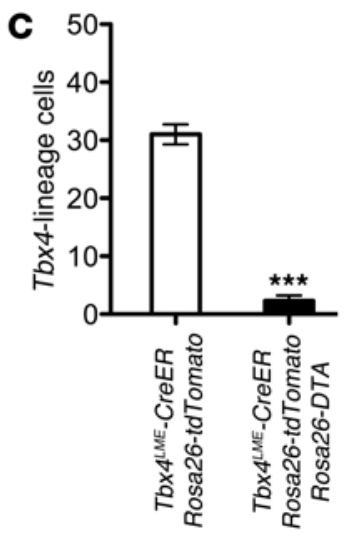
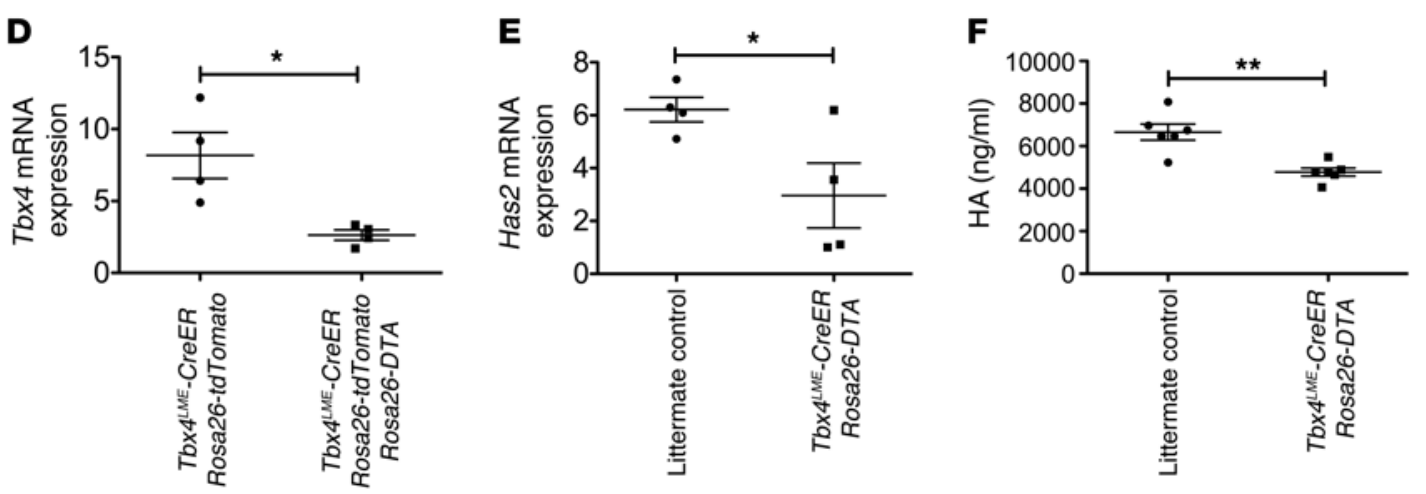

Figure 6. Ablation of TBX4 cells decreases HAS2 and HA expression. (A) Verification of ablation of Tbx4-lineage cells using Tbx4 $4^{L M E}$-CreER Rosa26tdTomato and Tbx4 ${ }^{\text {LME }}$-CreER Rosa26-tdTomato Rosa26-DTA mice. Five doses of tamoxifen were injected, and lungs were harvested 1 week later. (B) Representative images were taken from Tbx $4^{\text {LME }}$-CreER Rosa26-tdTomato and Tbx $4^{\text {LME }}$-CreER Rosa26-tdTomato Rosa26-DTA mouse lung ( $n=4$ lungs examined). (C) Quantification of Tbx4 lineage tracing and ablation, expressed as cells counted in B ( $n=4$ in each group of mice). (D and E) Tbx4 mRNA (D) and Has2 mRNA (E) expression of lung fibroblasts from Tbx4 $4^{L M E}$-CreER Rosa26-tdTomato and Tbx4 ${ }^{L M E}$-CreER Rosa26-tdTomato Rosa26-DTA were analyzed by quantitative RT-PCR, and normalized by GAPDH ( $n=4$ mice per group examined). (F) HA contents in conditioned media of lung fibroblasts from Tbx4 ${ }^{\text {LME }}$-CreER Rosa26-tdTomato and littermate controls were analyzed by HA ELISA. $n=6$ mice per group examined. ${ }^{*} P \leq 0.05,{ }^{* *} P \leq 0.01,{ }^{* * *} P \leq 0.001$ by 2-tailed Student's $t$ test, mean \pm SEM.

Conditional Tbx4 knockout in mesenchymal cells inhibits fibrosis. To directly demonstrate the role for TBX4 in lung fibrosis, we determined the impact of deletion of Tbx4 in either fibroblast or myofibroblast populations. Col1a2-CreER transgenic mice were crossed with $T b x 4^{f l / f l}$ mice (19), resulting in $T b x 4$ gene deletion in COL1 12 -expressing cells (Figure 8A). Five doses of tamoxifen were administered 1 week after bleomycin. Hydroxyproline content in Col1a2-CreER Tbx $4^{f / f l}$ mouse lungs was significantly decreased $(44 \%)$ in comparison with control mice 21 days after bleomycin administration (Figure 8B). Masson's trichrome and immunofluorescence staining of the lung for collagen, COL1 $\alpha 1$, and $\alpha$ SMA also showed similar results (Figure 8, C-E).

We then crossed $T b x 4^{f l / f l}$ mice with Acta2-CreER mice (referred to here as $\alpha$ SMA-CreER mice) in order to delete the Tbx4 gene in $\alpha$ SMA-expressing myofibroblasts upon tamoxifen administration (Figure 9A). Similar to the observation in COL1 $\alpha 2$-expressing cells, $\alpha$ SMA-CreER Tbx $4^{f l / f l}$ mouse lungs had reduced collagen content and $\alpha$ SMA expression compared with control mice 21 days after bleomycin by hydroxyproline assay ( $\sim 31 \%)$ and Masson's trichrome staining and immunofluorescence (Figure 9, C-E).

The lungs from the uninjured group of Col1a2-CreER Tbx $4^{f / f l}$ and $\alpha$ SMA-CreER Tbx $4^{f l f l}$ mice, which received tamoxifen injection to knock down the TBX4 expression, showed normal lung architecture (Supplemental Figure 12, A-G). The decreased expression of TBX4 in lung fibroblasts of the uninjured group of
Colla2-CreER Tbx $4^{f / f l}$ and $\alpha$ SMA-CreER Tbx $4^{f / f l}$ mice was verified (Supplemental Figure 12, H and K). Similar to TBX4 expression, the Has 2 mRNA expression and HA production of the lung fibroblasts were reduced (Supplemental Figure 12, L and M). These results indicate that the short-term $T b x 4$ gene deficiency under the static conditions does not affect lung structure, although the impact of long-term $T b x 4$ gene deficiency needs to be determined.

In contrast, bleomycin treatment of tamoxifen-exposed Ng2-CreER Tbx $4^{f l / f l}$ mice revealed that deletion of TBX4 expression in $\mathrm{NG}^{+}$cells had no impact on fibrosis following bleomycin treatment (Supplemental Figure 13, A and B), consistent with the data showing that NG2/tdT double-positive cells represent a minor population of $\alpha \mathrm{SMA}$-expressing cells (Supplemental Figure 2, B and C).

In order to determine a TBX4 role in myofibroblast transformation, collagen production, and fibroblast viability and apoptosis, we performed additional in vitro experiments. In IPF lung fibroblasts, the knockdown of Tbx4 by transfection of Tbx4 siRNA decreased $\alpha$ SMA protein expression by Western blot analysis (Supplemental Figure 14A; see supplemental material for full uncut gels). The secreted COL1 $\alpha 1$ production in IPF lung fibroblast supernatants was reduced in the presence and absence of TGF- $\beta 1$ treatment following Tbx4 knockdown (Supplemental Figure 14B). In regard to TBX4-mediated fibroblast viability, we tested the cell viability upon $T b x 4$ knockdown by Calcein AM cell viability assay. 
A TbX4 $4^{\text {LME }}$-CreER Rosa26-DTA

Bleo $2.5 \mathrm{U} / \mathrm{kg}$ Tam
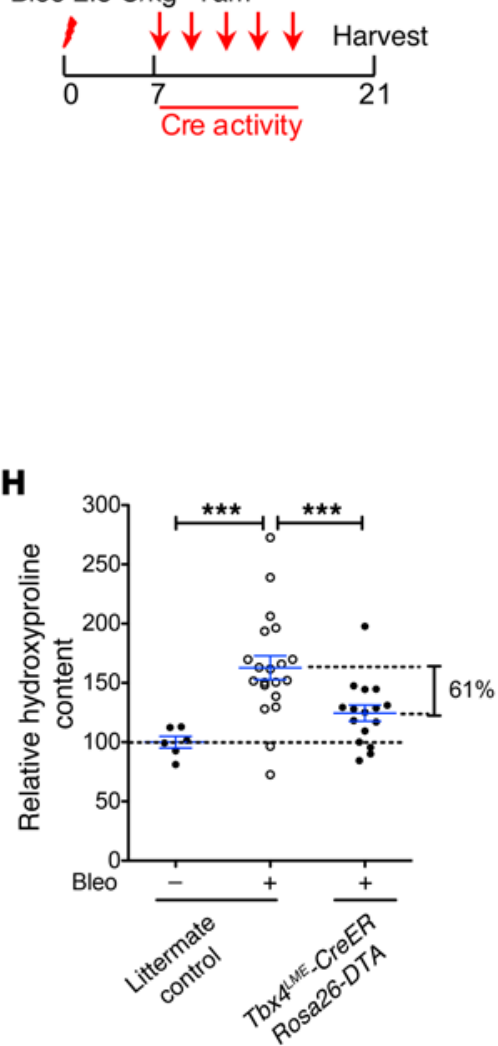

B

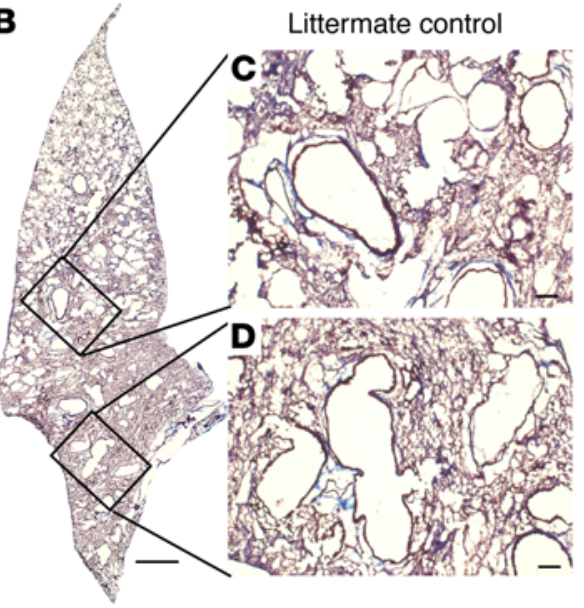

$\mathbf{E}$

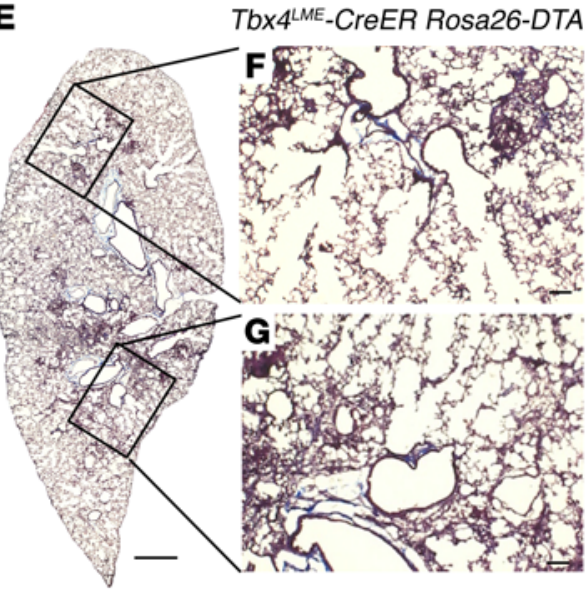

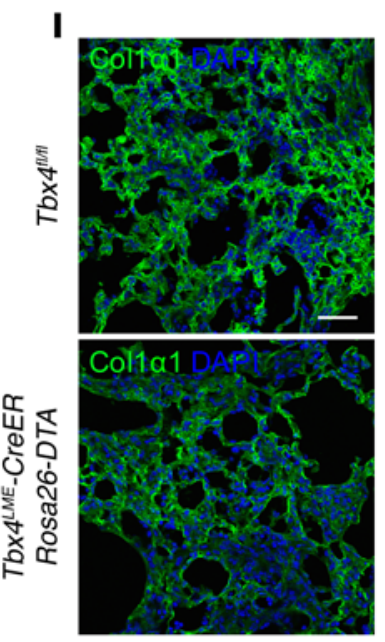
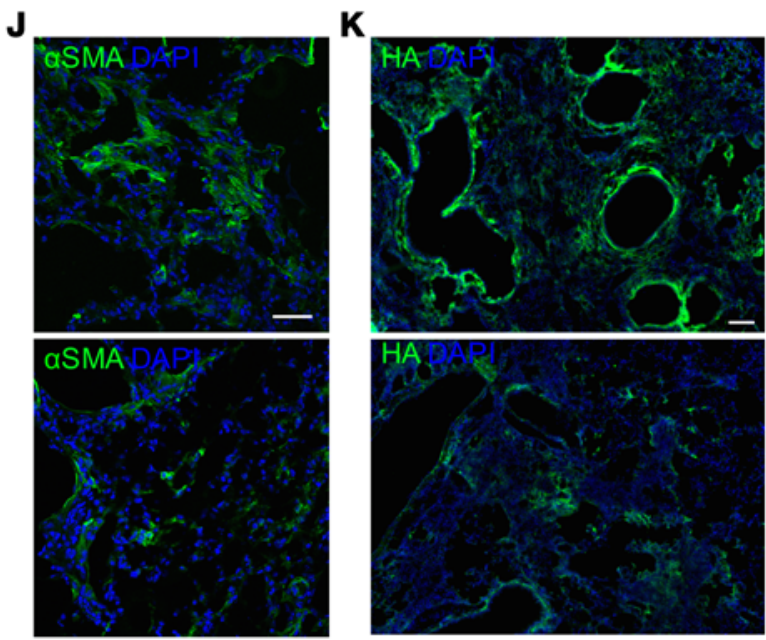

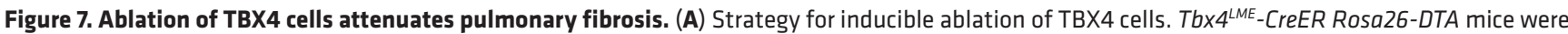
used in the subsequent experiment. The above-mentioned transgenic mice and their WT littermates (8-16 weeks old) were treated with bleomycin (2.5 $\mathrm{U} / \mathrm{kg}$ ), followed by 5 doses of tamoxifen $(0.2 \mathrm{mg} / \mathrm{g} /$ injection) every other day starting on $\mathrm{d} 7$. The lungs were collected for hydroxyproline content determination and Masson's trichrome staining on d21 after bleomycin. DTA, diphtheria toxin fragment A. (B-C) Representative Masson's trichrome staining images of bleomycin-treated (d21) adult littermate control (B-D) or Tbx $4^{\text {LME }}$-CreER Rosa26-DTA (E-C) mouse lung. (H) Targeting TBX4 cells reduced lung fibrosis (means $\pm \mathrm{SEM}$, ${ }^{* * *} P \leq 0.001$, 1-way ANOVA with Bonferroni test; uninjured littermate control, $n=6$; bleomycin [Bleo] littermate control, $n=20$;

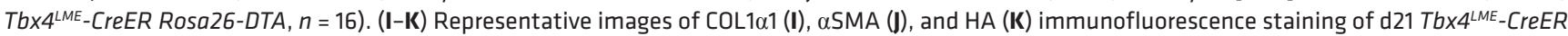
Rosa26-DTA mouse lung. $n=6$ mice per group examined. Scale bars: $1 \mathrm{~mm}$ (B and E) and $100 \mu \mathrm{m}(\mathbf{C}, \mathbf{D}, \mathbf{F}, \mathbf{G}$, and $\mathbf{I}-\mathbf{K})$.

The viability of Tbx4 knockdown cells was decreased as compared with control siRNA (Supplemental Figure 14C). In order to investigate whether loss of Tbx4 affects IPF lung fibroblast apoptosis, we performed a caspase- 3 activity assay. We found that upon transfection of Tbx4 siRNA, the caspase-3 activity in IPF lung fibroblasts was elevated in comparison with control siRNA.

Collectively, these data strongly suggest that TBX4 plays an important role in lung fibroblast invasion, SMA expression, collagen production, proliferation, and inhibition of apoptosis. Furthermore, TBX4 signaling drives fibrogenesis in bleomycininduced lung fibrosis in vivo.

$T B X 4$ regulates fibroblast invasion through HAS2. Our previous studies have shown that an invasive phenotype of fibroblasts was required for severe fibrosis. Fibroblasts isolated from lung tissue of IPF patients were more invasive than those from lung tissue of normal donors $(35,36)$. We next wanted to determine whether TBX4 plays a role in fibroblast invasion. Fibroblasts were isolated from Tbx $4^{L M E}$-Cre Rosa26-tdTomato mouse lungs and cultured in vitro for 3 passages, and the $\mathrm{tdT}^{+}$and $\mathrm{tdT}^{-}$cells were separated by flow cytometry. Immunofluorescence staining of $\mathrm{tdT}^{+}$ cells showed costaining with $\alpha \mathrm{SMA}$, COL1 $\alpha 1$, desmin, vimentin, PDGFR $\beta$, and NG2 (Supplemental Figure 15). Microarray analysis showed that $\mathrm{tdT}^{+}$cells had a more than 2-fold increase of $T b x 4$ mRNA expression (Figure 10A). Using the Matrigel invasion assay, we found that $T b x 4$-lineage cells were significantly more invasive than non-Tbx4-lineage cells (Figure 10B).

We previously determined that HAS2 regulates fibroblast invasion. To determine whether the increased invasiveness of Tbx4-lineage fibroblasts was due to enhanced HAS2 expression, we compared the expression levels of HAS2 between Tbx4-lineage and non-Tbx4-lineage fibroblasts, and found that Has 2 mRNA was increased in Tbx4-lineage fibroblasts compared with nonTbx4-lineage cells (Figure 10C and Supplemental Table 1). Furthermore, knocking down $T b x 4$ with a specific siRNA decreased 
A

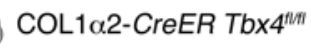



B $\quad T b \times 4^{n n}$

O COL1 $22-C r e E R$ Tbx4 ${ }^{n ! n}$
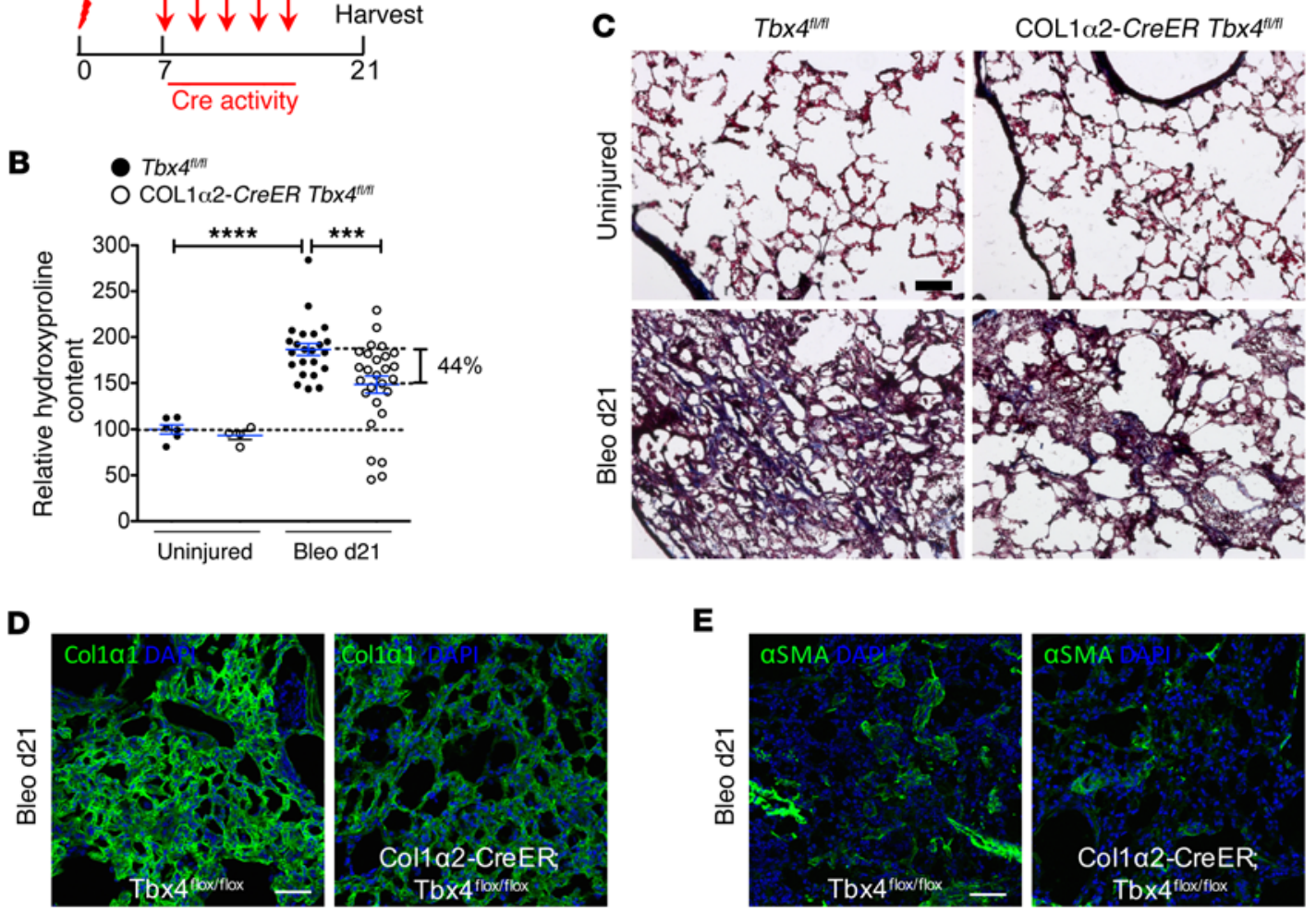

Figure 8. Loss of TBX4 expression in COL1 $\alpha 2$-expressing cells attenuates pulmonary fibrosis. (A) Strategy for inducible knockout of TBX4 expression in $\mathrm{COL}_{1 \alpha 2}{ }^{+}$cells. Col1a2-CreER Tbx4f/fl mice were used in these experiments. The above-mentioned mice and their WT littermates (8-16 weeks old) were treated with bleomycin $(2.5 \mathrm{U} / \mathrm{kg}$ ), followed by 5 doses of tamoxifen $(0.2 \mathrm{mg} / \mathrm{g} /$ injection) every other day starting on d7. The lungs were collected for hydroxyproline content determination on d21 after bleomycin. (B) Knockdown of Tbx4 in COL1a2-expressing cells decreased hydroxyproline content (means

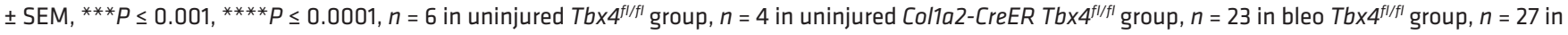
bleo Col1a2-CreER Tbx4//fl group). (C) Representative Masson's trichrome staining from lungs at d21 after bleomycin injection, showing decreased collagen

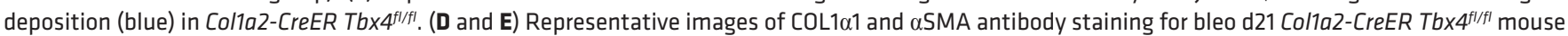
lung. $n=6$ mice per group examined. Scale bars: $100 \mu \mathrm{m}$ (C-E).

TGF- $\beta$-induced HA production in 3T3 mouse fibroblasts, suggesting that Has 2 is regulated by TBX4 (Figure 10D) in agreement with results from the allantois (37). There are 3 putative T-box response elements in the Has 2 promoter (37). Using a luciferase promoter assay, we showed that TBX4 could significantly induce the activity of vectors containing Has 2 promoters (Figure 10E), suggesting that TBX4 regulates Has 2 through transcriptional regulation.

In addition, we obtained MEF cells from CMV-Cre Tbx $4^{f / w}$ mice, designated $T b x 4^{K D}$ cells. Tbx 4 mRNA expression in $T b x 4^{K D}$ MEF cells was significantly less than that in control MEF cells (Figure 10F). TGF- $\beta$-induced Has 2 mRNA expression was decreased in $T b x 4^{K D}$ MEF cells (Figure 10G). HA production in Tbx $4^{K D}$ MEFs was dramatically reduced when treated with or without TGF- $\beta$ (Figure 10H). Furthermore, we found that the TBX4 gene expression was elevated in invasive human fibroblasts from IPF patients when compared with noninvasive human fibroblasts (Figure 10I). In lung fibroblasts from IPF patients, TBX4 siRNA transfection inhibited both invasiveness and TGF- $\beta$-induced HA production (Figure 10, J and K). Taken together, these data show that TBX4 mediates fibroblast invasion through HAS2.

\section{Discussion}

This study identifies a TBX4-driven fibrogenic cell population as a heterogeneous mesenchymal population, containing interstitial fibroblasts, vascular and airway smooth muscle cells, some pericytes, and a few endothelial cells in the adult mouse lung in normal and disease states. TBX4-labeled cells did not give rise to any lung cells expressing epithelial cell markers. More importantly, Tbx4lineage cells expanded, proliferated, and formed organized clonal patches during lung fibrosis in mice in vivo. Almost all of the $\alpha \mathrm{SMA}^{+}$ myofibroblasts and COL1 $\alpha 1^{+}$fibroblasts were Tbx4-derived cells, suggesting a critical role for this population of cells in lung fibrosis. This is further supported by data that lung fibrosis is significantly reduced by ablation of Tbx4-lineage cells. When Tbx4 was deleted in SMA- or COL1 $\alpha 2$-expressing fibroblasts, lung fibrosis was also significantly reduced. Thus several lines of evidence support a nonredundant role for TBX4 in regulating the pathobiology of lung fibrosis. We further showed that TBX4 mediated fibroblast invasiveness and HA production by transcriptional regulation of HAS2.

A major finding in this study was that virtually all $\alpha$ SMAexpressing myofibroblasts as well as COL1 $\alpha 1$-expressing fibro- 
A

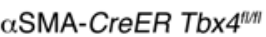
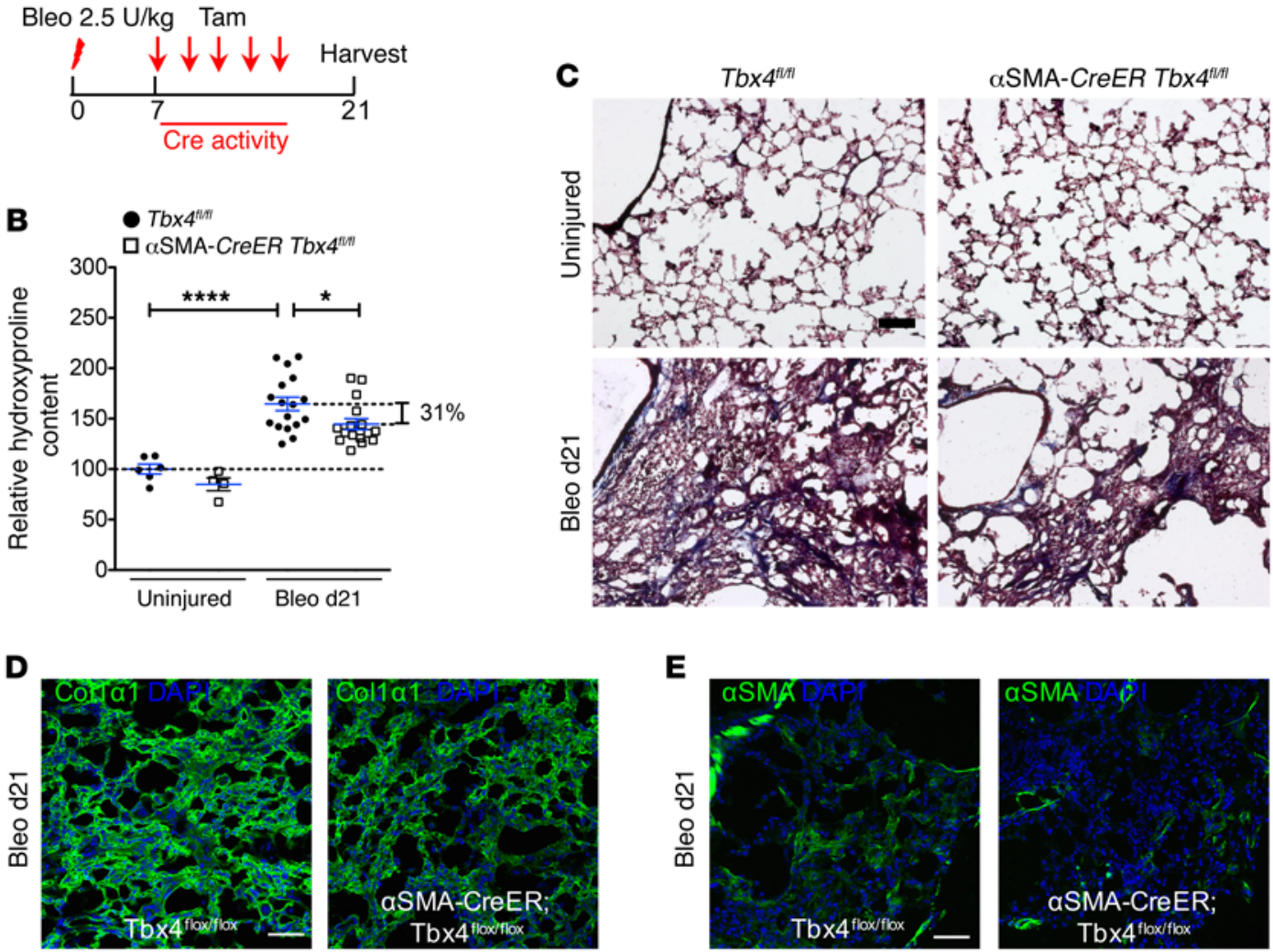

Figure 9. Inhibition of Tbx4 in $\alpha$ SMA+ cells attenuates lung fibrosis. (A) Strategy for inducible knockout of Tbx4 expression in $\alpha \mathrm{SMA}{ }^{+}$cells. Acta2-CreER $T b \times 4^{f / f l}$ mice were used in these experiments. The above-mentioned mice and their WT littermates (8-16 weeks old) were treated with bleomycin (2.5 U/ $\mathrm{kg})$, followed by 5 doses of tamoxifen $(0.2 \mathrm{mg} / \mathrm{g} /$ injection) every other day starting on $\mathrm{d} 7$. The lungs were collected for hydroxyproline content determination on d21 after bleomycin. (B) Knockdown of Tbx4 in $\alpha$ SMA-expressing cells decreased hydroxyproline content (means \pm SEM, ${ }^{*} P \leq 0.05$, ${ }^{* * * *} P \leq 0.0001$,

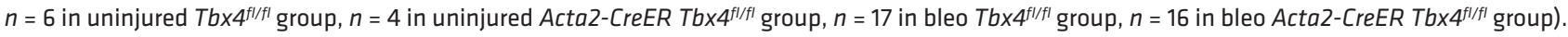
Hydroxyproline content values were expressed as percentage of control Tbx $4^{f / f l}$ group, which is set to $100 \%$. One-way ANOVA with Bonferroni test was used. (C) Representative Masson's trichrome staining from lungs at d21 after bleomycin injection, showing decreased collagen deposition (blue) in Acta2CreER Tbx $4^{f / / f l}$ mice. (D and E) Representative images of COL $1 \alpha 1$ and $\alpha S M A$ antibody staining for bleo d21 Acta2-CreER Tbx $4^{f / / f l}$ mouse lung. $n=6$ mice per group examined. Scale bars: $100 \mu \mathrm{m}(\mathbf{C}-\mathbf{E})$.

blasts were derived from TBX4-expressing cells. These data suggest that within the lung, a local lineage of interstitial resident fibroblasts promote fibrogenesis. The observation is consistent with recent studies in skin fibrosis $(2,38)$, lung fibrosis $(3)$, and liver fibrosis (33). This appears to be in contrast to kidney fibrosis, where only $50 \%$ of myofibroblasts were suggested to arise from resident fibroblasts (4). The demonstration that $T b \times 4$-lineage cells are devoid of all epithelial cell markers is in line with previous work (5) suggesting that cell lineage commitment does not cross between epithelial and stromal compartments in lung fibrosis. Epithelial-mesenchymal transition (EMT) has been suggested in the pathogenesis of cardiac fibrosis (39), lung fibrosis $(13,14,40,41)$, and kidney fibrosis $(42,43)$. Although our study did not directly examine the role of EMT in lung fibrosis, the data did not support a major role for EMT in lung fibrosis. In addition, extrapulmonary contribution of fibroblasts and myofibroblasts is not regulated by Tbx4-derived cells. Bone marrow-derived stromal progenitor cells such as fibrocytes $(44)$ have been suggested in lung fibrosis $(9,45)$. A very small fraction of Tbx4-derived cells were observed in the bone marrow. Collectively, these data suggest that the majority of myofibroblasts and fibroblasts that accumulate during fibrosis are $T b \times 4$ lineage-derived mesenchymal intrapulmonary cells regardless of the particular cell surface expression marker.

A critical goal for the field of tissue fibrosis is to define fibroblast origins under homeostatic and disease states. Current views on the origins of fibroblasts during development and in diseases have been established mostly based on antibody staining, rather than the use of transgenic reporter markers or systematic quantitative analysis of fibroblast lineages present in the lung. A few recent studies have attempted to genetically trace the origin of myofibroblasts during tissue fibrosis. For example, resident perivascular Adam12- and Gli1-lineage cells, and Foxd1-lineage pericytes, stained positive for aSMA antibody after tissue injury $(2,3,17)$. Among them, Foxd1-lineage pericytes coexpress Col1a1 reporter GFP (3). Furthermore, Engrailed 1-derived cells are positive for collagen type I antibody staining in skin fibrosis (38). In the current study, we revealed that $T b x 4$-lineage cells constitutively labeled from the embryonic stage or inducibly labeled postnatally 
A

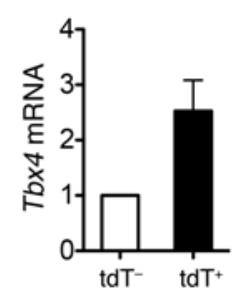

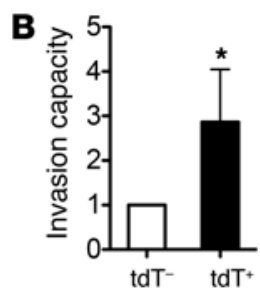

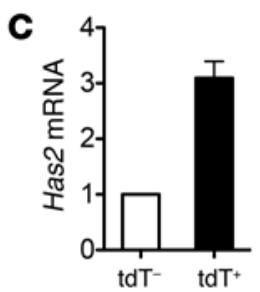

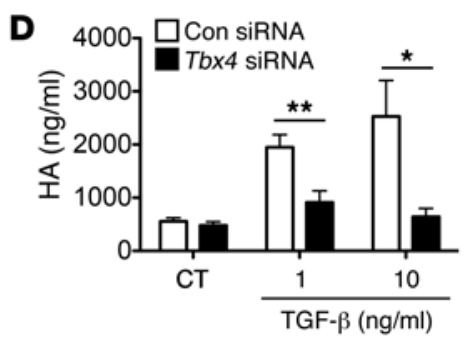

\section{E}

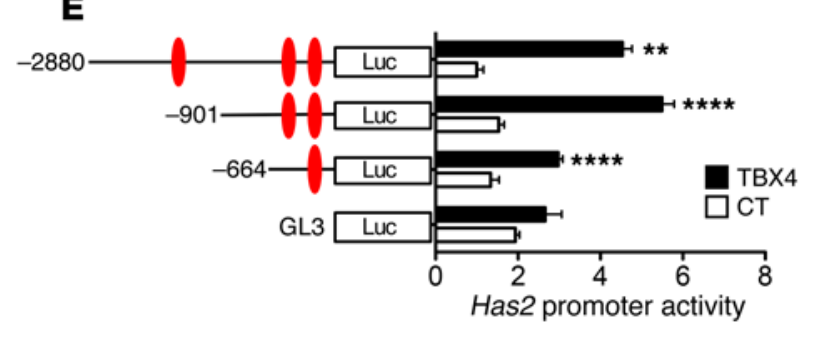

H

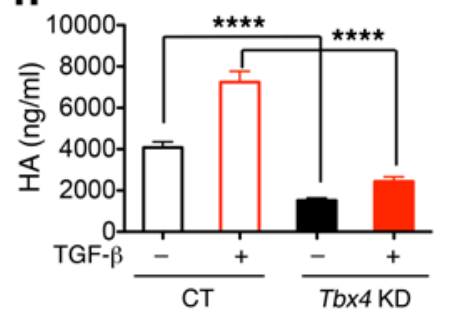

I

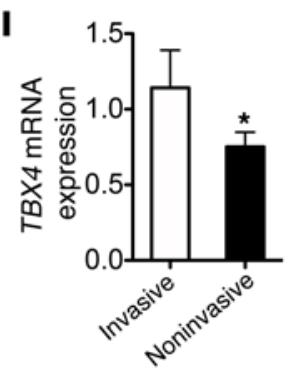

$\mathbf{F}$

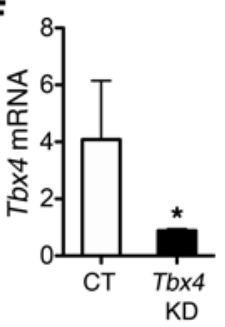

G

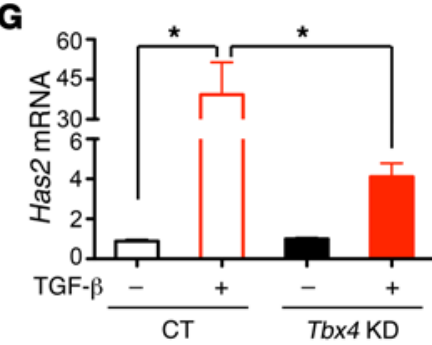

J

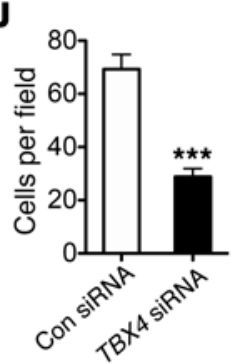

K

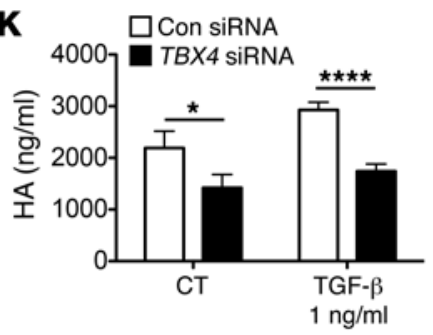

Figure 10. TBX4 regulates fibroblast invasion through regulation of Has2. (A) $T b x 4$ mRNA expression was increased in sorted tdT ${ }^{+}$cells from $T b x 4-C r e^{T_{g}}$ Rosa26-tdTomato mice. (B) tdT ${ }^{+}$fibroblasts were more invasive. Invasion capacity values were expressed as relative fold change of control, which is set to 1. (C) Has2 mRNA level was elevated in tdT' fibroblasts. (D) Tbx4 siRNA reduced TCF- $\beta 1$-induced HA release by mouse fibroblast $3 T 3$ cells. Cells were transfected with either Tbx4 siRNA or control siRNA (Con). Forty-eight hours later cells were treated with TCF- $\beta 1$ at $1 \mathrm{ng} / \mathrm{ml}$ or $10 \mathrm{ng} / \mathrm{ml}$ concentration for 24 hours. HA was measured in conditioned media. CT, control. (E) TBX4 enhanced Has2 promoter activity. All Has2 promoter constructs showed increased activity over the promoterless vector pGL3 when cotransfected with a TBX4-expressing vector in HEK 293 cells. (F-H) MEF cells were isolated from E14 embryo of CMV-Cre Tbx $4^{f / w}$ and WT mice. MEF cells at passage 3 were used for experiments. (F) Tbx4 mRNA levels were compared between Tbx4-deficient and WT MEFs. (G) MEF cells were treated with or without TGF- $\beta 1$ ( $5 \mathrm{ng} / \mathrm{ml}$ ) overnight. Has2 mRNA expression was analyzed using RT-PCR. (H) HA in supernatants was measured with HA ELISA. (I) TBX4 gene expression was elevated in invasive human fibroblasts from IPF patients. (J) Invasion assay for IPF fibroblasts was performed 48 hours after transfection of control and TBX4 siRNA. Knocking down TBX4 with siRNA reduced the invasiveness of IPF fibroblasts. (K) Knocking down TBX4 with siRNA reduced HA production of primary fibroblasts from IPF patients with or without $1 \mathrm{ng} / \mathrm{ml}$ TGF- $\beta 1$ treatment after 48 hours. $n=4$ in each group were analyzed. All results are the mean of triplicate experiments $\pm S E M .{ }^{*} P \leq 0.05,{ }^{* *} P \leq 0.01,{ }^{* * *} P \leq 0.001$, ${ }^{* * * *} P \leq 0.0001$, as analyzed by 2-tailed Student's $t$ test (B, F, I, and J) or 1-way ANOVA with Bonferroni test (D, E, G, H, and $\left.\mathbf{K}\right)$.

can differentiate into heterogeneous stromal cell types including $\alpha \mathrm{SMA}^{+}$and COL1 $\alpha 1^{+}$cells by antibody staining. To confirm our antibody staining results, we used several transgenic reporter lines, including $\alpha$ SMA-GFP and COL1 $\alpha 1-G F P$ mice, to detect the colocalization of aSMA- and Col1a1-expressing cells within the Tbx4-lineage cells by means of confocal microscopy and FACS analysis. Our observations showed that aSMA-expressing cells are dramatically increased in the fibrotic lung. Almost all of the aSMA-expressing cells (over 90\%) are Tbx4-lineage cells. We also detected a large portion of $T b x 4$-lineage-only ( $\mathrm{TBX}^{+} \alpha \mathrm{SMA}^{-}$) cells, indicating that not only $\alpha \mathrm{SMA}^{+}$cells but other non- $\alpha \mathrm{SMA}$ mesenchymal cells accumulate during fibrosis. This is in line with a previous study showing that multiple stromal populations contribute to pulmonary fibrosis, including $\alpha$ SMA, vimentin, desmin, PDGFR $\beta$, and NG2, by using antibody staining (5). In addition, we showed that the vast majority of COL1 $\alpha 1^{+}$cells are $T b x 4$-lineage cells. COL1 $11^{+}$cells are considered resident fibroblasts $(3,17,33)$, while some data suggest that circulating fibrocytes can express COL1 $11(44,46)$. The data presented in this study suggest that the majority of COL1 $\alpha^{+}$cells are resident fibroblasts, although a small proportion of COL1 $\alpha 1$ single-positive cells, the circulating fibrocytes, were observed.

Pericytes are defined as mesenchymal cells that share a common basement membrane with endothelial cells (47). They have been suggested as 1 of the sources of fibroblasts during tissue fibrosis $(17,28)$. Our lineage labeling data demonstrate that a maximum of $20 \%$ of NG2+ cells are Tbx4-lineage cells. Considering that the majority of $\alpha \mathrm{SMA}^{+}$cells are $\mathrm{Tb} x 4$-lineage cells, our results suggest that $\mathrm{NG}^{+}$cells represent a minor population of $\alpha \mathrm{SMA}^{+}$cells. This observation is consistent with a previous study using $\mathrm{Ng} 2$-CreER mice, which demonstrated that $\mathrm{NG}^{+}$pericytes do not express high levels of $\alpha$ SMA in fibrotic lungs (5). These also 
support recent reports that NG2 may not be a reliable marker for all pericytes $(3,4,48)$. Antibody staining with another pericyte marker, PDGFR $\beta$, showed much more colocalization with Tbx4lineage cells than NG2 staining. This suggested that PDGFR $\beta$ may be a better marker for pericytes than NG2. Collectively, these data suggest that $\mathrm{NG}^{+}$pericytes are not the major contributor to myofibroblasts in lung fibrosis.

Tbx4-lineage progenitor cells not only contain $\alpha \mathrm{SMA}^{+}$, $\mathrm{COL} 1 \alpha 1^{+}$, and $\mathrm{NG}^{+}$cells, as we have demonstrated with transgenic gene reporter mice, but also include a few endothelial cells by antibody staining. This population does not increase during fibrosis, consistent with a report that PECAM-1 costained with Tbx4-lineage cells in embryonic mouse lung (26). It has been reported that mesenchymal-endothelial transition contributes to neovascularization during heart injury (49). As TBX4 is expressed early in lung mesenchyme, the data suggest that mesenchymalto-endothelial transition might occur during development. It would be interesting to use additional lineage tracing tools to determine when Tbx4-lineage cells start to express endothelial cell markers. Whether a portion of mesenchymal cells differentiate to endothelial cells or a small portion of endothelial cells require TBX 4 signaling is unknown.

We used mesenchyme-specific clonal analysis (22) to examine expansion of TBX4-expressing cells during fibrosis. We found that TBX4-expressing cells expanded clonally. Within 3 weeks after bleomycin injury, clone size can be as many as 6 cells. Furthermore, these patches seemed to be monoclonal, indicating that regeneration foci are derived from a single $T b x 4$ progenitor. These data suggest that the mesenchymal progenitors during fibrogenesis are similar to the embryonic-stage mesenchymal cells, which are highly proliferative and progressively expand (22). The adult mesenchymal progenitors are unlike the alveolar stem cells, which undergo long-term self-renewal and diffuse proliferation $(50,51)$. These findings suggest that mesenchymal progenitors proliferate and self-renew during lung fibrogenesis. These data support the previously suggested concept that during lung fibrosis, embryonic gene programs are reactivated (52). However, more comprehensive clonal analysis of $T b x 4$ progenitor cells such as long-term tracing, single dose versus multiple doses of tamoxifen, before and after bleomycin injury, and coexpression with other mesenchymal/lineage markers is needed to further delineate the clonal nature of these cells.

As a mesenchymal transcription factor, TBX4 could control downstream gene expression and impact key fibroblast effector functions. In order to gain insights into possible target genes, we analyzed Tbx4-lineage and non-Tbx4-lineage cell gene expression, and identified mesenchymal genes that could be regulated by TBX4. Among them, Has 2 is upregulated in TBX4-expressing cells. Has 2 is 1 of the 3 HA synthase genes that have been identified. We have previously shown that fibroblasts from Acta2-Has2 transgenic mice (referred to here as $\alpha \mathrm{SMA}-\mathrm{Has} 2$ mice) are more invasive, while fibroblasts from Fsp1-Cre Has $2^{f / f l}$ mice (also known as S100a4-Cre Has $2^{f l / f l}$ mice) had diminished invasive capacity (35). $\alpha$ SMA-Has 2 mice showed increased fibrosis after bleomycin injury, and mice with conditional deletion of Has 2 in mesenchymal cells exhibited less fibrosis (35). We then analyzed how TBX4 regulates Has 2 and impacts the fibroblast invasive phenotype. We found that TBX4 regulates both fibroblast invasiveness and HA production. TBX4 binds to the Has 2 promoter and triggers Has 2 gene expression. These data establish TBX4 as a proximal regulator of Has 2 expression, HA production, and fibroblast invasiveness. Interestingly, the array data suggest that TBX4 may regulate additional fibroblast and ECM remodeling genes, including Fgf, Pdgfr, Mmp, and Adam family genes. It has been shown that TBX4 and TBX5 are linked to the activity of FGF, BMP, and WNT signaling pathways that are required for limb outgrowth and patterning (53). TBX4 and TBX5 are critical for expression of mesenchymal FGF10 in embryonic mouse lung (54). It has been reported that TBX4 and TBX5 trigger limb formation by the induction of both $W n t$ and $F g f(55)$. There are no reports to date directly linking $T b x 4$ and $M m p$ gene expression, although inhibition of the T-box transcription factor Brachyury showed downregulation of Mmp2 and Mmp24 in the context of EMT in cancer (56). Moreover, T-BET, another T-box transcription factor, was found increased together with MMP3 when TGF- $\beta$ was blocked in human gut (57). Further investigations into the interrelationships between TBX4 and matrix remodeling genes are warranted.

An intriguing possibility is raised of a hierarchical relationship of TBX4-lineage fibroblasts with other mesenchymal populations, such as FGF10 ${ }^{+}, \mathrm{GLI}^{+}$, $\mathrm{ADAM}_{2}{ }^{+}, \mathrm{PDGFR}^{+}$, and FOXD1 ${ }^{+}$ cells. TBX4 is a potential upstream regulator of these genes and is detected as early as E9.25 exclusively in lung mesenchyme without epithelial expression $(18,20,22,24,58,59)$. TBX4 induces mesenchymal genes and the pluripotent stem cell-related transcription factors (Gata2 and Gata3; Smad1, Smad2, Smad3, and Smad5; and Snail) in adult mice, suggesting that TBX4 can be a critical driver of fibroblast differentiation. Furthermore, the identification of TBX4 as a regulator of Has 2 expression points to this pathway as a potential target for therapeutic intervention.

Collectively, these data demonstrate that Tbx4-lineage cells represent most of the resident mesenchymal progenitors, expand during fibrosis, and form clonal patches. TBX4 drives fibroblast matrix production and invasiveness through the regulation of Has2. Further understanding the origin, signaling partners, and functions of Tbx4-derived mesenchymal cells could provide new insights into the pathogenesis of progressive fibrotic diseases.

\section{Methods}

Mice. Tbx 4 lung enhancer-Cre transgenic mice (Tbx $4^{L M E}$-Cre or Tbx4$\left.C r e^{T g}\right)$ and $T b x 4^{L M E_{-}} C r e E R$ transgenic mice were recently described (22). Tbx4-Cre knock-in (Tbx4-Cre $\left.{ }^{K i}\right)$ mice were reported (24). Tbx4Cre Rosa26-tdTomato mouse lines were obtained by crossing of Tbx4-Cre knock-in (Tbx4-Cre $\left.{ }^{K i}\right)$ mice or transgenic mice with lineage reporter Rosa26-tdTomato mice (25) (Brigid L.M. Hogan, Duke University, Durham, NC). To obtain Tbx4 $4^{L M E}$-CreER Rosa26tdTomato, Tbx $4^{\mathrm{LME}_{-}}$CreER mice were crossed to Rosa26-tdTomato mice. Tbx $4^{L M E}$-CreER Confetti mice were obtained by crossing of $T b x 4^{L M E}$-CreER mice with Rosa26 Confetti mice (34) (Jackson Laboratory). Triple-heterozygous $\alpha$ SMA-GFP Tbx4-Cre Rosa26-tdTomato mice were created by crossing of Tbx4-Cre Rosa26-tdTomato mice with Acta2-GFP mice (referred to here as $\alpha$ SMA-GFP mice) (60) (Robert N. Fariss, National Eye Institute, Bethesda, MD). Triple-heterozygous Tbx4-Cre Rosa26-tdTomato mice were bred with Col1a1-GFP mice (referred to here as COL1 $\alpha 1-$ GFP mice) (33) (David A. Brenner, 
University of California, San Diego, La Jolla, CA) to generate COL1 $\alpha 1-$ GFP Tbx4-Cre Rosa26-tdTomato. Tbx4-Cre Rosa26-tdTomato mice were bred with Ng2-YFP mice (4) (Raghu Kalluri, University of Texas MD Anderson Cancer Center, Houston, TX) to create the Ng2-YFP Tbx4-Cre Rosa26-tdTomato triple-heterozygous mice. Tbx $4^{L M E}$-CreER mice were bred with Rosa26-DTA mice (61) (Jackson Laboratory) to generate $T b x 4^{L M E}$-CreER Rosa26-DTA mice.

$T b x 4^{f l / f l}$ mice were described previously (20). Acta2-CreER mice (referred to here as $\alpha$ SMA-CreER mice) (62) were crossed with $T b x 4^{f / f l}$ mice to create the $\alpha \mathrm{SMA}-\mathrm{CreER} T b x 4^{f / / f l}$ mice. Col1 $\alpha 2-C r e E R T b x 4^{f / / l}$ mice were generated by breeding of Colla2-CreER mice (referred to here as COL1 $\alpha 2-C r e E R$ mice) (63) (Jackson Laboratory) with $T b x 4^{f / f l}$ mice. Conditional NG2 cell-specific Tbx4 knockout mice (Ng2-CreER $T b x 4^{f / f l}$ mice) were generated by breeding of Ng2-CreER (also known as Cspg4-CreER) mice (64) (Jackson Laboratory) with $T b x 4^{f / f l}$ mice. CMV-Cre mice were from Jackson Laboratory. Tamoxifen (SigmaAldrich) dissolved in corn oil was injected i.p. at 1-5 doses of $0.1 \mu \mathrm{g} / \mathrm{g}$ or $0.2 \mu \mathrm{g} / \mathrm{g}$ body weight to induce Cre-mediated recombination. All mice were on a C57BL/6 background.

Murine lung fibrosis model. Mice (both male and female), 8 to 16 weeks old, were subjected to bleomycin-induced lung injury $(35,36)$. Bleomycin at $2.5 \mathrm{U} / \mathrm{kg}$ was injected intratracheally. Mouse lungs were harvested on day 14 or day 21 for histology, hydroxyproline assay, and single-cell isolation.

Histology, immunofluorescence, and confocal imaging. Immunofluorescent staining on frozen sections $(5 \mu \mathrm{m}$ or $12 \mu \mathrm{m})$ was performed using primary antibodies to $\alpha$ SMA (catalog A2547, clone 1A4, SigmaAldrich), desmin (catalog RB9014-P0, Thermo Scientific), NG2 (cata$\log$ AB5320, Millipore), vimentin (catalog sc-7557, Santa Cruz Biotechnology), Col1 $\alpha 1$ (catalog NB600-408, Novus Biologicals), PDGFR $\beta$ (catalog 04-397, Millipore), vWF (catalog Ab6994, Abcam), lipid acid (Vybrant DiO Cell-Labeling Solution, catalog v22886, Life Technologies), SPC (catalog AB3786, Millipore), Scgb1a1 (a gift of the Barry Stripp laboratory, Cedars-Sinai Medical Center, Los Angeles, CA), T1 (clone 8.1.1-c, Developmental Studies Hybridoma Bank), BrdU (cata$\log$ OBT0030, Accurate Chemical and Scientific), biotinylated recombinant human aggrecan (staining for HA, custom order from R\&D Systems Inc., lot PQO051505A), and associated Alexa Flour 488-conjugated secondary antibodies (Life Technologies) per manufacturer instructions. RFP was stained with paraffin lung sections (catalog 600-401-379, Rockland). Stained sections were imaged using a Zeiss 780 reverse Laser Scanning Confocal Microscope.

BrdU incorporation assay. Cell proliferation was quantified by BrdU labeling. Briefly, BrdU (5 mg/ml, catalog B-9285, Sigma-Aldrich) was injected i.p. into bleomycin-treated Tbx $4^{L M E}$-CreER Rosa26-tdTomato mice at a dose of $10 \mu \mathrm{l} / \mathrm{g}$ body weight at day 21. After 3 hours, lungs were collected and fixed by $10 \%$ formaldehyde. Lungs were embedded with OCT compound (Sakura Finetek). Frozen sections underwent antigen retrieval by application of $1.5 \mathrm{~N} \mathrm{HCl}$ to the section for 30-45 minutes. Sections were then processed for immunofluorescent staining with anti-BrdU antibody (1:250; catalog OBT0030, Accurate Chemical and Scientific), followed by Alexa Fluor 488-conjugated secondary antibody (1:500; Invitrogen).

Scale processing and imaging. Tbx $4^{L M E}-C r e E R$ Confetti mouse lungs were fixed with $10 \%$ formalin for 4-16 hours and washed with PBS overnight. Tissues were transferred to Scale A2 and kept on a rocker at $4^{\circ} \mathrm{C}$ for $1-2$ weeks until maximal tissue clearing occurred. Whole-mount samples immersed in Scale A2 solution were imaged on the inverted Zeiss 780 confocal microscope in a 35 -mm glass-bottom microwell dish with $\mathrm{a} \times 20$ objective. $\mathrm{Z}$-stack and tiled images were acquired from large areas and volumes of tissue. Images were processed by ZEN software.

Hydroxyproline assay. Collagen content in lung tissue was measured with the conventional hydroxyproline method (65). The ability of the assay to completely hydrolyze and recover hydroxyproline from collagen was confirmed using samples containing known amounts of purified collagen.

Fibroblast isolation and culture. Primary fibroblasts were derived from mouse lungs as described previously (66). The mouse primary fibroblasts were used from 3 to 6 passages. Human lung fibroblasts were isolated from lung transplant explants obtained from patients with IPF as described previously $(35,66,67)$. All cases fulfilled multidisciplinary diagnostic criteria described in the American Thoracic Society/European Respiratory Society consensus statement. In addition, pathological confirmation was obtained for every case. IPF was confirmed by the identification of a usual interstitial pneumonia under the light microscope. Patient demographics were as follows: 2 females and 2 males; ages between 51 and 78 with median age 67; percentage forced vital capacity (FVC\%) 51-78, median 57; and percentage diffusing capacity factor of the lung for carbon monoxide (DLCO\%) 18-42, median 30.75 . Samples of whole lung tissue were obtained at the time during orthotopic lung transplantation surgery. Samples were immediately processed following removal from the body. The specimens were cut into small pieces ( $\sim 1 \mathrm{~mm}$ in diameter), and cultured in DMEM supplemented with $15 \%$ FBS, $100 \mathrm{U} / \mathrm{ml}$ penicillin, $100 \mu \mathrm{g} / \mathrm{ml}$ streptomycin, $5 \mu \mathrm{g} / \mathrm{ml}$ gentamicin, and $0.25 \mu \mathrm{g} / \mathrm{ml}$ amphotericin B. The cells of passages $4-8$ were used for invasion assay, siRNA interference assays, and COL1 $\alpha 1$ and HA amount measurements. Four cell lines were analyzed per group.

Matrigel invasion assay. The invasive behavior of Tbx4-lineage and non-Tbx4-lineage fibroblasts isolated from Tbx4-Cre Rosa26$t d$ Tomato mouse lungs was analyzed essentially as described previously (35). Equal numbers of fibroblasts $\left(5 \times 10^{4}\right)$ in $500 \mu \mathrm{l}$ of $10 \% \mathrm{FBS}$ complete medium were plated onto the BioCoat Matrigel Invasion Chamber (BD Biosciences). Seven hundred fifty microliters of $10 \%$ FBS complete medium with $10 \mathrm{ng} / \mathrm{ml}$ PDGF (120-HD-001, R\&D Systems Inc.) was added to the bottoms of wells. After 24 hours of incubation in $\mathrm{CO}_{2}$ incubator, media were removed, and the polycarbonate filters with the invaded cells were washed once with PBS followed by fixing and staining with the Protocol Hema 3 stain set. Matrigel matrix and noninvading cells on the upper surface of the filter were removed by wiping with a cotton swab, and the filters were removed from the insert by a scalpel blade and were mounted onto glass slides. The invading cells of each sample were counted in 9 randomly selected fields of duplicate filters under a microscope at $\times 400$ magnification. Tbx 4 siRNA effects on 3T3 cell line and human IPF lung fibroblast invasion were assessed using $30 \mathrm{nM}$ siRNA. The cells were transfected with siRNAs for 48 hours before the invasion assay was performed.

$M E F$ isolation and culture. Mouse embryonic fibroblast cells were derived from E14.5 embryos of CMV-Cre Tbx $4^{f / w}$ or control (CMVCre only or $T b x 4^{f / w}$ only) mice as described previously (68). The cells were used from 3 to 6 generations. MEFs were treated with $5 \mathrm{ng} / \mathrm{ml}$ TGF- $\beta 1$ overnight. RNA was isolated for RT-PCR, and supernatant was collected for HA ELISA.

Flow cytometry. Mouse lung or bone marrow was isolated from Tbx $4^{L M E_{-}}$Cre Rosa26-tdTomato, aSMA-GFP Tbx $4^{L M E_{-}}$Cre Rosa26- 
tdTomato, Col1a1-GFP Tbx $4^{L M E}$-Cre Rosa26-tdTomato, and NG2-YFP Tbx $4^{L M E}$-Cre Rosa26-tdTomato mice after perfusion. Single mouse lung cells were dissociated using a mouse lung dissociation kit and gentleMACS dissociator (Miltenyi Biotec). Single bone marrow cells from $T b x 4^{L M E}$-Cre Rosa26-tdTomato mice were dissociated with a pipette, followed by rbc lysis. Cells were analyzed with Fortessa (BD Biosciences). Fluorescence intensity is expressed in arbitrary units on a logarithmic scale or on a linear scale for forward scatter.

Cultured passage 3 fibroblasts from untreated $T b x 4^{L M E_{-}}$Cre Rosa26-tdTomato mice were sorted for $\mathrm{tdT}^{+}$and $\mathrm{tdT}^{-}$cells with FACS Aria 3 (BD Biosciences) to 95\%-98\% purity.

Quantitative real-time PCR. Real-time RT-PCR was used to quantify the relative mRNA levels of Has 2 in CMV-Cre Tbx $4^{f / w}$ or control MEF cells with or without human recombinant TGF- $\beta 1$ treatment using gene-specific primers. In brief, total RNA was purified using RNAqueous-4PCR kit (Life Technologies) and was reversed to cDNA using High-Capacity cDNA Reverse Transcription Kit (Life Technologies) according to the manufacturer's instructions. Has 2 gene levels in the resultant cDNAs were examined using the ABI 7500 Fast Detection system (Applied Biosystems) with SYBR Green as fluorescent dye enabling real-time detection of PCR products according to the manufacturer's protocol (Power SYBR Green PCR Master Mix, Applied Biosystems). The relative expression levels of the gene were determined against Gapdh levels in the samples. The following primers were used: mouse Has2 (GenBank accession no. NM_008216) forward, 5'-ACGACGACCTTTACATGATGGA-3'; mouse Has2 reverse, 5'-GATGTACGTGGCCGATTTGCT-3'; mouse Tbx4 (GenBank accession no. NM_011536.2) forward, 5'-TCACTGGATGCGGCAGTTGGTCTCT-3'; mouse Tbx4 reverse, 5'-CACGTGGGTGCAAAAGGCTGTGTTT-3'; mouse Gapdh forward, 5'-ATCATCTCCGCCCCTTCTG-3'; and mouse Gapdh reverse, 5'-GGTCATGAGCCCTTCCACAAC-3'.

Affymetrix cDNA microarray. Cultured passage 3 fibroblasts from (untreated) $T b x 4^{L M E}$-Cre Rosa26-tdTomato mice were sorted for $\mathrm{tdT}^{+}$ and tdT cells with FACS Aria 3 (BD Biosciences) to 95\%-98\% purity. Total RNA was assessed for quality with Agilent 2100 Bioanalyzer G2939A (Agilent Technologies) and Nanodrop 8000 spectrophotometer (Thermo Scientific/Nanodrop). Hybridization targets were prepared with the Ovation Pico WT Amp V2 kit (NuGen) and the Encore Biotin Module (NuGen) from total RNA, hybridized to GeneChip Mouse 4302.0 arrays in Affymetrix GeneChip hybridization oven 645, washed in Affymetrix GeneChip Fluidics Station 450, and scanned with Affymetrix GeneChip Scanner $7 \mathrm{G}$ according to standard Affymetrix GeneChip Hybridization, Wash, and Stain protocols.

Data were background-subtracted and normalized within the array using the LOESS normalization by GeneSpring GX 11 software. The complete microarray data set is available at the National Center for Biotechnology Information Gene Expression Omnibus (http:// www.ncbi.nlm.nih.gov/geo/) under accession number GSE68427.

HA quantification. The HA contents in cultured media of human lung fibroblasts, 3T3 cell lines, and MEF cells were quantified using the Hyaluronan DuoSet ELISA (DY3614, R\&D Systems Inc.).

RNA interference. Tbx4 siRNA effects on 3T3 cell line and human IPF lung fibroblast HA production were assessed with siRNA specific to Tbx4 (mTbx4 siRNA: 5'-rGrCrArCrUrGrCrCrArArGrArArArCrArUrGrGrArArArGGT-3'; hTbx4 siRNA: 5'-rUrGrCrArArUrUrArUrCrUrArArGrArArGrUrGrArCrUrUTG-3'; Universal scrambled negative control siRNA duplex, SR30004, OriGene Technologies).
The cells were transfected with $30 \mathrm{nM}$ siRNAs for 48 hours, and then treated with $5 \mathrm{mg} / \mathrm{ml}$ TGF- $\beta 1$ overnight. The supernatants were collected thereafter for HA measurement. Invasion assays were done using cells transfected for 48 hours.

Promoter luciferase assay. The 2,880-bp Has2 promoter luciferase reporter plasmid was provided by Chin Chiang (69) (Vanderbilt University Medical Center, Nashville, TN) and contains sequence from -2,326 to exon 1 of Has 2 in pGL3 Basic plasmid (Promega). The 901-bp (chromosome 15, NC_000081.6: -436 to exon 1) and 664-bp (-112 to exon 1) Has 2 promoter luciferase reporter plasmid was generated by PCR and cloned upstream of pGL3 Basic plasmid. Primers used were as follows: forward 1: 5'-CAAGCTCGAGGGAATCCTTGTAACG-3'; forward 2: 5'-CCGCCTCGAGTCCCGCCCAGTCCCT-3'; and reverse: 5'-TGCCAAGCTTCTTGTTCAGCTCCTGCTCATAGA-3'. CMV promoterdriven GFP-tagged TBX4 expression plasmid was purchased from GeneCopoeia. Luciferase assays were performed essentially as previously (69), using the Promega Dual luciferase Reporter assay system. To determine the effect of TBX4 on different lengths of the Has 2 promoter activity, HEK 293 cells were cotransfected with Has 2 promoter or pGL3 Basic plasmid and TBX4-expressing plasmid for 48 hours prior to the luciferase assay. All reporter assays were normalized using Renilla luciferase as an internal control. Each data point represents the mean of triplicate wells with error bar representing the SEM.

Calculation of the internal surface of a lung. To ensure consistent and reproducible results, all (left and right) lungs were fixed by $10 \%$ formalin and with the same pressure, degassed, and embedded in OCT. We performed step sectioning on each block of lung. For the slides of each step section, more than 5 lung images were sampled randomly under Zeiss AXIO microscopy ( $\times 10$ objective). Lung morphometric analysis was performed on the images of the lung parenchymal area for uninjured and injured lung without severe fibrotic lesion. Mean linear intercept, also known as mean chord length (70), was calculated by point and intersection counting using a grid line system (71).

Western blot. To detect $\alpha$ SMA and $\beta$-actin expression, $25 \mu \mathrm{g}$ of cell lysate was separated through $10 \%$ SDS-PAGE electrophoresis, blotted onto a nitrocellulose membrane (Life Technologies), and incubated with mAbs against $\alpha$ SMA (1:1,000; F3777, Sigma Chemical Co.) and $\beta$-actin (1:1,000; 12620, Cell Signaling Technology). The bands were visualized using the ECL system (Bio-Rad).

Collagen $1 \alpha 1$ ELISA. The collagen $1 \alpha 1$ contents in cultured media of human lung fibroblasts were quantified using a collagen $1 \alpha 1$ direct ELISA developed in the laboratory. Standard curve for the assay was generated using collagen type I solution (C3867, Sigma-Aldrich) and collagen $1 \alpha 1$ antibody (ab21286, Abcam). The concentration of the collagen $1 \alpha 1$ in the cell supernatants was determined by extrapolation from the appropriate standard curve.

Caspase-3 activity assay. IPF fibroblasts were cultured on 96-well plates. Caspase- 3 activity assay was performed 48 hours after Tbx 4 siRNA transfection. The amount of protein in cell lysates was determined using a BCA protein assay kit (Pierce Chemical Co.). Caspase3/ CPP32 Fluorometric Assay Kit (BioVision) was used to examine the caspase-3 activity assay.

Calcein AM cell viability assay. The cell viability of IPF fibroblasts 48 hours after Tbx4 siRNA transfection was analyzed by Calcein AM Cell Viability Assay Kit (Trevigen).

Statistics. Data are expressed as the mean \pm SEM. Differences in measured variables between experimental and control groups were 
assessed by Student's $t$ tests (2-tailed) or Wilcoxon rank-sum test with nonparametric data. One-way ANOVA with Bonferroni test was used for multiple comparisons. The survival curves were compared using the log-rank test. Results were considered statistically significant at $P \leq 0.05$. GraphPad Prism software was used for statistical analysis.

Study approval. All human lung fibroblast experiments were approved by the Cedars-Sinai Medical Center IRB and were in accordance with the guidelines outlined by the board. Informed consent was obtained from each subject (IRB: Pro00032727).

All mouse studies were approved by the IACUC at Cedars-Sinai Medical Center (protocols IACUC004722 and IACUC004751).

\section{Author contributions}

PWN, DJ, TX, and JL conceived and designed the study. TX performed most of the in vivo fate-mapping and in vitro experiments. JL, NL, and $\mathrm{CH}$ participated in in vivo experiments. JL, YZ, and WL participated in culture experiments. MK, DM, PC, VEP, BRS, $\mathrm{RX}$, and JD provided critical reagents, intellectual discussions, and interpretation of data. TX, JL, DJ, and PWN analyzed data and wrote the paper. All authors discussed the data. DJ and PWN approved the submission of the manuscript.

\section{Acknowledgments}

We thank Brigid L.M. Hogan for Rosa26-tdTomato mice, Robert Fariss for $\alpha$ SMA-GFP mice, David Brenner for COL1 $\alpha 1-$ GFP mice, and Raghu Kalluri for NG2-YFP mice. We thank Mark Krasnow for suggestions during the initiation of the project. We thank Chin Chiang (Vanderbilt University Medical Center) for pHas2 luciferase plasmids. We thank Patricia Lin and Laura Dieu at the Cedars-Sinai Medical Center Flow Cytometry Core for cell sorting. We thank Laura-Leigh Rowlette and Zhengzheng Wei (Duke University) for microarray analysis. This work was supported by NIH grants P01-HL108793, R01-HL060539, and AI052201 (to P.W. Noble), R01-HL122068 (to D. Jiang), and R37H-D033082 (to V.E. Papaioannou), and a grant from California Institute for Regenerative Medicine (RB5-07302 to P.W. Noble).

Address correspondence to: Paul W. Noble or Dianhua Jiang, Women's Guild Lung Institute, Department of Medicine, Cedars-Sinai Medical Center, 8700 Beverly Boulevard, Los Angeles, California 90048, USA. Phone: 310.423.1888; E-mail: paul.noble@cshs. org (P.W. Noble). Phone: 310.423.3176; E-mail: dianhua.jiang@ cshs.org (D. Jiang).
1. Noble PW, Barkauskas CE, Jiang D. Pulmonary fibrosis: patterns and perpetrators. J Clin Invest. 2012;122(8):2756-2762.

2. Dulauroy S, Di Carlo SE, Langa F, Eberl G, Peduto L. Lineage tracing and genetic ablation of ADAM12(+) perivascular cells identify a major source of profibrotic cells during acute tissue injury. Nat Med. 2012;18(8):1262-1270.

3. Hung C, et al. Role of lung pericytes and resident fibroblasts in the pathogenesis of pulmonary fibrosis. Am J Respir Crit Care Med. 2013;188(7):820-830

4. LeBleu VS, et al. Origin and function of myofibroblasts in kidney fibrosis. Nat Med. 2013;19(8):1047-1053.

5 . Rock JR, et al. Multiple stromal populations contribute to pulmonary fibrosis without evidence for epithelial to mesenchymal transition. Proc Natl Acad Sci U S A. 2011;108(52):E1475-E1483.

6. Jordana M, et al. Heterogeneous proliferative characteristics of human adult lung fibroblast lines and clonally derived fibroblasts from control and fibrotic tissue. Am Rev Respir Dis. 1988;137(3):579-584.

7. Zeisberg M, Kalluri R. Cellular mechanisms of tissue fibrosis. 1. Common and organ-specific mechanisms associated with tissue fibrosis. Am J Physiol Cell Physiol. 2013;304(3):C216-C225.

8. Krenning G, Zeisberg EM, Kalluri R. The origin of fibroblasts and mechanism of cardiac fibrosis. JCell Physiol. 2010;225(3):631-637.

9. Hashimoto N, Jin H, Liu T, Chensue SW, Phan SH. Bone marrow-derived progenitor cells in pulmonary fibrosis. J Clin Invest. 2004;113(2):243-252.

10. Andersson-Sjöland A, et al. Fibrocytes are a potential source of lung fibroblasts in idiopathic pulmonary fibrosis. Int J Biochem Cell Biol. 2008;40(10):2129-2140.

11. Phillips RJ, et al. Circulating fibrocytes traffic to the lungs in response to CXCL12 and mediate fibrosis. J Clin Invest. 2004;114(3):438-446.
12. Degryse AL, et al. Repetitive intratracheal bleomycin models several features of idiopathic pulmonary fibrosis. Am J Physiol Lung Cell Mol Physiol. 2010;299(4):L442-L452.

13. Kim KK, et al. Alveolar epithelial cell mesenchymal transition develops in vivo during pulmonary fibrosis and is regulated by the extracellular matrix. Proc Natl Acad Sci U S A. 2006;103(35):13180-13185.

14. Tanjore $\mathrm{H}$, et al. Contribution of epithelial-derived fibroblasts to bleomycin-induced lung fibrosis. Am J Respir Crit Care Med. 2009;180(7):657-665.

15. Hashimoto N, et al. Endothelial-mesenchymal transition in bleomycin-induced pulmonary fibrosis. Am J Respir Cell Mol Biol. 2010;43(2):161-172.

16. Li Z, Jimenez SA. Protein kinase $\mathrm{C} \delta$ and c-Abl kinase are required for transforming growth factor $\beta$ induction of endothelial-mesenchymal transition in vitro. Arthritis Rheum. 2011;63(8):2473-2483.

17. Kramann R, et al. Perivascular Gli1+ progenitors are key contributors to injury-induced organ fibrosis. Cell Stem Cell. 2015;16(1):51-66.

18. Takeuchi JK, et al. Tbx5 and Tbx4 genes determine the wing/leg identity of limb buds. Nature. 1999;398(6730):810-814.

19. Naiche LA, Papaioannou VE. Tbx4 is not required for hindlimb identity or post-bud hindlimb outgrowth. Development. 2007;134(1):93-103.

20. Arora R, Metzger RJ, Papaioannou VE. Multiple roles and interactions of Tbx 4 and Tbx 5 in development of the respiratory system. PLoS Genet. 2012;8(8):e1002866.

21. Du J, et al. Different expression patterns of Gli1-3 in mouse embryonic maxillofacial development. Acta Histochem. 2012;114(6):620-625.

22. Kumar ME, Bogard PE, Espinoza FH, Menke DB, Kingsley DM, Krasnow MA. Mesenchymal cells. Defining a mesenchymal progenitor niche at single-cell resolution. Science. 2014;346(6211):1258810.
23. Luria V, Krawchuk D, Jessell TM, Laufer E, Kania A. Specification of motor axon trajectory by ephrin-B:EphB signaling: symmetrical control of axonal patterning in the developing limb. Neuron. 2008;60(6):1039-1053.

24. Naiche LA, Arora R, Kania A, Lewandoski M, Papaioannou VE. Identity and fate of Tbx4-expressing cells reveal developmental cell fate decisions in the allantois, limb, and external genitalia. Dev Dyn. 2011;240(10):2290-2300.

25. Madisen L, et al. A robust and high-throughput Cre reporting and characterization system for the whole mouse brain. Nat Neurosci. 2010;13(1):133-140.

26. Zhang W, et al. Spatial-temporal targeting of lung-specific mesenchyme by a Tbx 4 enhancer. BMC Biol. 2013;11:111.

27. Greif DM, et al. Radial construction of an arterial wall. Dev Cell. 2012;23(3):482-493.

28. Humphreys BD, et al. Fate tracing reveals the pericyte and not epithelial origin of myofibroblasts in kidney fibrosis. Am J Pathol. 2010;176(1):85-97.

29. Lin SL, Kisseleva T, Brenner DA, Duffield JS. Pericytes and perivascular fibroblasts are the primary source of collagen-producing cells in obstructive fibrosis of the kidney. Am J Pathol. 2008;173(6):1617-1627.

30. Border WA, Noble NA. Transforming growth factor beta in tissue fibrosis. $N$ Engl J Med. 1994;331(19):1286-1292.

31. Hoyles RK, et al. An essential role for resident fibroblasts in experimental lung fibrosis is defined by lineage-specific deletion of high-affinity type II transforming growth factor $\beta$ receptor. Am J Respir Crit Care Med. 2011;183(2):249-261.

32. Luo Y, et al. A novel profibrotic mechanism mediated by TGF $\beta$-stimulated collagen prolyl hydroxylase expression in fibrotic lung mesenchymal cells. J Pathol. 2015;236(3):384-394.

33. Iwaisako $\mathrm{K}$, et al. Origin of myofibroblasts in the fibrotic liver in mice. Proc Natl Acad Sci US A. 2014;111(32):E3297-E3305. 
34. Livet J, et al. Transgenic strategies for combinatorial expression of fluorescent proteins in the nervous system. Nature. 2007;450(7166):56-62.

35. Li Y, et al. Severe lung fibrosis requires an invasive fibroblast phenotype regulated by hyaluronan and CD44. J Exp Med.2011;208(7):1459-1471.

36. Lovgren AK, et al. $\beta$-arrestin deficiency protects against pulmonary fibrosis in mice and prevents fibroblast invasion of extracellular matrix. Sci Transl Med.2011;3(74):74ra23.

37. Arora R, del Alcazar CM, Morrisey EE, Naiche LA, Papaioannou VE. Candidate gene approach identifies multiple genes and signaling pathways downstream of Tbx 4 in the developing allantois. PLoS One. 2012;7(8):e43581.

38. Rinkevich Y, et al. Skin fibrosis. Identification and isolation of a dermal lineage with intrinsic fibrogenic potential. Science. 2015;348(6232):aaa2151

39. Kalluri R, Neilson EG. Epithelial-mesenchymal transition and its implications for fibrosis. JClin Invest. 2003;112(12):1776-1784.

40. Chapman HA. Epithelial-mesenchymal interactions in pulmonary fibrosis. Annu Rev Physiol. 2011;73:413-435.

41. Willis BC, et al. Induction of epithelial-mesenchymal transition in alveolar epithelial cells by transforming growth factor- $\beta 1$ : potential role in idiopathic pulmonary fibrosis. Am J Pathol. 2005;166(5):1321-1332.

42. Zeisberg M, et al. BMP-7 counteracts TGF-beta1induced epithelial-to-mesenchymal transition and reverses chronic renal injury. Nat Med. 2003;9(7):964-968.

43. Iwano M, Plieth D, Danoff TM, Xue C, Okada $\mathrm{H}$, Neilson EG. Evidence that fibroblasts derive from epithelium during tissue fibrosis. J Clin Invest. 2002;110(3):341-350.

44. Bucala R, Spiegel LA, Chesney J, Hogan M, Cera$\mathrm{mi}$ A. Circulating fibrocytes define a new leukocyte subpopulation that mediates tissue repair. Mol Med.1994;1(1):71-81.

45. Moore BB, et al. CCR2-mediated recruitment of fibrocytes to the alveolar space after fibrotic injury. Am J Pathol. 2005;166(3):675-684.

46. van Deventer HW, Palmieri DA, Wu QP, McCook EC, Serody JS. Circulating fibrocytes prepare the lung for cancer metastasis by recruiting Ly-6C+ monocytes via CCL2.J Immunol. 2013;190(9):4861-4867.

47. Bergers G, Song S. The role of pericytes in blood-vessel formation and maintenance. Neurooncology. 2005;7(4):452-464.

48. Duffield JS. Cellular and molecular mechanisms in kidney fibrosis. J Clin Invest. 2014;124(6):2299-2306.

49. Ubil E, et al. Mesenchymal-endothelial transition contributes to cardiac neovascularization. Nature. 2014;514(7524):585-590.

50. Messier B, Leblond CP. Cell proliferation and migration as revealed by radioautography after injection of thymidine- $\mathrm{H} 3$ into male rats and mice. Am J Anat. 1960;106:247-285.

51. Barkauskas CE, et al. Type 2 alveolar cells are stem cells in adult lung.J Clin Invest. 2013;123(7):3025-3036.

52. Selman M, Pardo A, Kaminski N. Idiopathic pulmonary fibrosis: aberrant recapitulation of developmental programs?. PLoS Med. 2008;5(3):e62.

53. Rodriguez-Esteban C, Tsukui T, Yonei S, Magallon J, Tamura K, Izpisua Belmonte JC. The T-box genes Tbx 4 and Tbx 5 regulate limb outgrowth and identity. Nature. 1999;398(6730):814-818.

54. Cebra-Thomas JA, Bromer J, Gardner R, Lam GK, Sheipe H, Gilbert SF. T-box gene products are required for mesenchymal induction of epithelial branching in the embryonic mouse lung. Dev Dyn. 2003;226(1):82-90.

55. Takeuchi JK, Koshiba-Takeuchi K, Suzuki T, Kamimura M, Ogura K, Ogura T. Tbx5 and Tbx4 trigger limb initiation through activation of the Wnt/Fgf signaling cascade. Development. 2003;130(12):2729-2739.

56. Fernando RI, Litzinger M, Trono P, Hamilton DH, Schlom J, Palena C. The T-box transcription factor Brachyury promotes epithelial-mesenchymal transition in human tumor cells. JClin Invest. 2010;120(2):533-544.

57. Di Sabatino A, et al. Blockade of transforming growth factor beta upregulates T-box transcription factor T-bet, and increases $\mathrm{T}$ helper cell type 1 cytokine and matrix metalloproteinase- 3 production in the human gut mucosa. Gut. 2008;57(5):605-612.

58. Naiche LA, Papaioannou VE. Loss of Tbx4 blocks hindlimb development and affects vascularization and fusion of the allantois. Development. 2003;130(12):2681-2693.

59. Sakiyama J, Yamagishi A, Kuroiwa A. Tbx4-Fgf10 system controls lung bud formation during chicken embryonic development. Development. 2003;130(7):1225-1234.

60. Condren AB, et al. Perivascular mural cells of the mouse choroid demonstrate morphological diversity that is correlated to vasoregulatory function. PLoS One. 2013;8(1):e53386.

61. Voehringer D, Liang HE, Locksley RM. Homeostasis and effector function of lymphopenia-induced "memory-like" $\mathrm{T}$ cells in constitutively T cell-depleted mice. J Immunol. 2008;180(7):4742-4753.

62. Wendling O, Bornert JM, Chambon P, Metzger D. Efficient temporally-controlled targeted mutagenesis in smooth muscle cells of the adult mouse. Genesis. 2009;47(1):14-18.

63. Zheng B, Zhang Z, Black CM, de Crombrugghe B, Denton CP. Ligand-dependent genetic recombination in fibroblasts: a potentially powerful technique for investigating gene function in fibrosis. Am J Pathol. 2002;160(5):1609-1617.

64. Zhu X, Hill RA, Dietrich D, Komitova M, Suzuki $R$, Nishiyama A. Age-dependent fate and lineage restriction of single NG2 cells. Development. 2011;138(4):745-753.

65. Adamson IY, Bowden DH. The pathogenesis of bleomycin-induced pulmonary fibrosis in mice. Am J Pathol. 1974;77(2):185-197.

66. Jiang $\mathrm{D}$, et al. Inhibition of pulmonary fibrosis in mice by CXCL10 requires glycosaminoglycan binding and syndecan-4. JClin Invest. 2010;120(6):2049-2057.

67. Meltzer EB, et al. Bayesian probit regression model for the diagnosis of pulmonary fibrosis: proof-of-principle. BMC Med Genomics. 2011;4:70.

68. Jozefczuk J, Drews K, Adjaye J. Preparation of mouse embryonic fibroblast cells suitable for culturing human embryonic and induced pluripotent stem cells. J Vis Exp. 2012;(64):3854.

69. Liu J, Li Q, Kuehn MR, Litingtung Y, Vokes SA, Chiang C. Sonic hedgehog signaling directly targets Hyaluronic Acid Synthase 2, an essential regulator of phalangeal joint patterning. Dev Biol. 2013;375(2):160-171.

70. Campbell H, Tomkeieff SI. Calculation of the internal surface of a lung. Nature. 1952;170(4316):116-117.

71. Foronjy RF, Mercer BA, Maxfield MW, Powell CA, D’Armiento J, Okada Y. Structural emphysema does not correlate with lung compliance: lessons from the mouse smoking model. Exp 